\title{
əOn the Formation Mechanism of Cirrus Banding: Radiosonde Observations, Numerical Simulations, and Stability Analyses
}

\author{
KAZUYA YAMAZAKI ${ }^{\mathrm{a}}$ AND HiROAKI MiURA ${ }^{\mathrm{a}}$ \\ ${ }^{a}$ Department of Earth and Planetary Science, Graduate School of Science, The University of Tokyo, Tokyo, Japan
}

(Manuscript received 22 November 2020, in final form 17 July 2021)

\begin{abstract}
The structures and formation mechanisms of cirrus banding are investigated by analyzing radiosonde observations, conducting high-resolution numerical experiments, and performing linear stability analyses. In all 29 cases of cirrus bands that were analyzed, radiosonde observational data indicate that statically unstable layers exist. The detected banding clouds were aligned nearly parallel to the vertical shear vector in the unstable layer. In highresolution numerical experiments using the cloud-resolving Scalable Computing for the Advanced Library and Environment Regional Model (SCALE-RM), cirrus bands forming in the outflow layer of a tropical cyclone are explicitly simulated. The existence of statically unstable layers and band-parallel background vertical wind shear are commonly identified in the simulations. Sensitivity experiments and heat budget analyses demonstrated that the unstable stratification within the cirrus clouds was maintained by the cloud-radiation interactions. To reveal the behavior of fluid instabilities in the cirrus bands, linear stability analyses in a basic state constructed from the radiosonde observations were performed. The fastest-growing disturbance is highly similar to that of the previously known thermal-shear instability in a uniform and isolated unstable layer and the results obtained by radiosonde observations and numerical simulations. All of the results consistently indicate that thermal-shear instability is responsible for the formation of cirrus banding. Our results not only follow previous modeling studies but also provide observational support, quantification of the destabilization by the cloud-radiation interactions, and a theoretical basis of the thermal-shear instability in a complex environment near cirrus bands.
\end{abstract}

KEYWORDS: Cirrus clouds; Stability; Radiosonde/rawinsonde observations; Satellite observations; Nonhydrostatic models

\section{Introduction}

Transverse cirrus banding is defined in the Glossary of Meteorology (Glickman 2000) as "irregularly spaced bandlike cirrus clouds that form nearly perpendicular to a jet stream axis." Similar banding structures have been identified near tropical cyclones (TCs; Zehr 2004) and mesoscale convective systems (MCSs; Lenz et al. 2009; Trier et al. 2010). Cirrus bands accompanying those disturbances are sometimes called "radial bands" because of their radial alignment from the center of the disturbances (Trier et al. 2010). In this study, these bands are collectively referred to as "cirrus bands," regardless of their origin. Those banding clouds are sometimes accompanied by harmful aviation turbulence (Ellrod 1985; Lenz et al. 2009), indicating the importance of understanding the nature of the cirrus banding.

As cirrus bands often form under strong vertical and horizontal shears, multiple instabilities have been suggested as possible formation mechanisms (Knox et al. 2010). On the one hand, Ohno and Miura (1982) conducted linear stability analyses of the dry Boussinesq equations and identified growing billows resulting

\footnotetext{
D Denotes content that is immediately available upon publication as open access.
}

Corresponding author: Kazuya Yamazaki, yamazaki@eps.s.utokyo.ac.jp from the Kelvin-Helmholtz instability (KHI), which they concluded is responsible for the formation of cirrus banding. They used empirical profiles of a jet stream, where a weakly stable layer existed but no unstably stratified layer was present. On the other hand, Trier et al. (2010) investigated cirrus bands accompanying an MCS via numerical experiments and found statically unstable layers within cirrus bands, indicating that the radial cirrus bands within the outer MCS anvil are manifestations of the thermalshear instability. In cloudy layers, they used the moist BruntVäisälä frequency $\left(N_{m}^{2}\right)$ defined by Durran and Klemp (1982) instead of the dry buoyancy frequency $\left(N^{2}\right)$, and found moist statically unstable layers that satisfy $N_{m}^{2}<0$. Similar modeling studies have been carried out for an extratropical cyclone (Kim et al. 2014) and a convectively enhanced jet stream (Trier and Sharman 2016), where smaller-scale billow clouds associated with the KHI are also identified.

Although not focused on cirrus bands, observational studies on such low-stability layers within outflow regions of TCs have been conducted by Molinari et al. (2014). They used data from dropsonde observations around TCs and detected the frequent appearance of regions with low bulk Richardson numbers. They categorized such turbulent layers in three groups, one of which resides in the central dense overcast and features near-zero static stability. Duran and Molinari (2016) further investigated such turbulent layers using rawinsonde observations, and documented their characteristics about spatial distribution, sensitivity to cyclone intensity, and diurnal cycle. While these layers with nearzero static stability seem closely related to cirrus bands around 
TCs, this relation remains unclear because they did not focus on banding structures of clouds. Thus, to the best of our knowledge, neither the weakly stable stratification assumed by Ohno and Miura (1982) nor the statically unstable layer identified in simulations by Trier et al. (2010) has been verified within cirrus bands via observations.

Furthermore, the behavior of the thermal-shear instability near the cirrus banding has not been fully understood yet. The theory of the thermal-shear instability applied by Trier et al. (2010) was based on an isolated layer with a uniform value of $N^{2}$ and vertical wind shear (Hill 1968; Asai 1970). In reality, statically unstable layers coexist with neighboring stable layers; their dynamic interaction can potentially modify the nature of the thermal-shear instability. This type of complex interaction within environmental conditions typical for cirrus bands has not yet been fully investigated.

In this study, observational, numerical, and theoretical analyses were carried out to advance our knowledge of the remaining issues. First, we observationally investigated the thermal, wind, and geometric structures of cirrus bands by analyzing data from high-resolution radiosondes and lowEarth-orbit (LEO) satellites (section 2). Next, a TC simulation was conducted in an idealized setup using a cloud-resolving model to illustrate the structures and heat budgets of cirrus bands in anvil clouds (section 3). Using the thermal and wind structures obtained from the observations and simulations, linear stability analyses were performed to explore the nature of growing disturbances, with moist effects taken into account implicitly (section 4 ). These results are discussed in section 5, and a summary of this work is presented in section 6 .

\section{Observations}

To obtain observational evidence of the static stability within cirrus bands and their horizontal structures, analyses of radiosonde data and LEO satellite images were conducted.

\section{a. Radiosonde observations}

We use data from routine radiosonde observations (Kizu et al. 2018) conducted by the Japan Meteorological Agency (JMA). Although previous studies have observationally investigated low-stability layers within TC outflow regions (Molinari et al. 2014; Duran and Molinari 2016), the vertical scale of unstable layers in cirrus bands remains unclear because they did not focus specifically on cirrus bands. Thus, to capture instabilities in vertically thin layers as well as thick ones, our analyses exclusively target data having high vertical resolution disseminated since 5 July 2018. This dataset contains temperatures, dewpoint temperatures with respect to liquid water, wind speeds, wind directions, longitudes, and latitudes at each observation level, which are vertically spaced approximately every $7 \mathrm{~m}$. Observations are carried out every day at 0000 and 1200 UTC at observation sites listed in Table 1. Radiosonde-observed cases of cirrus bands from 5 July 2018 to 31 July 2020 were combined with 10.3$\mu \mathrm{m}$ infrared images obtained by Himawari-8, a geostationary satellite operated by JMA (Bessho et al. 2016). The
TABLE 1. Geographical locations of radiosonde observation sites used in this study.

\begin{tabular}{lcc}
\hline \multicolumn{1}{c}{ Site name } & Lat $\left({ }^{\circ} \mathrm{N}\right)$ & Lon $\left({ }^{\circ} \mathrm{E}\right)$ \\
\hline Sapporo & 43.0 & 141.7 \\
Kushiro & 43.0 & 144.4 \\
Akita & 39.7 & 140.1 \\
Wajima & 37.4 & 136.9 \\
Tateno & 36.1 & 140.1 \\
Hachijojima & 33.1 & 139.8 \\
Matsue & 35.5 & 135.8 \\
Shionomisaki & 33.5 & 135.8 \\
Fukuoka & 33.6 & 130.4 \\
Kagoshima & 31.6 & 130.5 \\
Naze & 28.4 & 129.6 \\
Minami-daitojima & 25.8 & 131.2 \\
Minami-torishima & 24.3 & 154.0 \\
\hline
\end{tabular}

case extraction is performed based on three conditions: (i) a radiosonde penetrates a region featuring cirrus bands, (ii) cirrus bands are vivid enough to determine their azimuth direction with little ambiguity, and (iii) temperature and wind profiles do not exhibit questionable fluctuations which could be artifacts. Owing to difficulties in establishing objective criteria for these conditions, extraction is performed manually. Recently, a deep learning technique for detecting cirrus bands from RGB true color composite images was developed by Miller et al. (2018). While it is currently unclear whether their method can be directly applied to the infrared images that we use, it can potentially provide a viable criterion for condition (i) in future studies. In this study, 29 cases summarized in Table 2 met our subjective criteria and were all used for the analyses below. Four cases (1-2, 1-4, 1-17, and 1-20) were from TC outflows, and the others were associated with jet streams or extratropical cyclones.

As the adiabatic lapse rate in a saturated condition is different from that in an unsaturated one, the accurate discrimination between the two is essential for an accurate diagnosis of the stability. Here, we use the relative humidity with respect to ice $\left(\mathrm{RH}_{i}\right)$ because cloud particles comprising cirrus bands are mostly frozen. However, the appropriate threshold for the cloud detection is nontrivial because clouds are sometimes subsaturated by up to $20 \%$ (Korolev and Isaac 2006). Additionally, in the upper troposphere, measurements of the humidity using radiosondes are often subject to large errors. For example, Jaubert et al. (1999) noted that the ratio of questionable $\mathrm{RH}$ measurements can be $10 \%$ or more from 350 to $50 \mathrm{hPa}$. As a remedy to tackle those difficulties, we additionally use the satellite-based estimation of the cloud-top height by the High-Resolution Cloud Analysis Index (Suzue et al. 2016) derived from Himawari-8 images to detect cloudy layers. For example, in case 1-20, the satellite-estimated cloud top is approximately at $14 \mathrm{~km} \mathrm{MSL}$, where $\mathrm{RH}_{i}$ from the radiosonde observation is around $80 \%$ (Fig. 1). Thus, in this study, layers which are roughly above the $350-\mathrm{hPa}$ level and have an $\mathrm{RH}_{i}$ roughly above $80 \%$ were subjectively assumed to be saturated, as 
TABLE 2. Dates and observation sites of radiosonde observations used in this study. The geographical locations of the observation sites are given in Table 1.

\begin{tabular}{lllcll}
\hline \hline Case ID & \multicolumn{1}{c}{ Time and date } & Observation site & Case ID & Time and date & Observation site \\
\hline $1-1$ & 0000 UTC 22 Jul 2018 & Kushiro & $1-16$ & 1200 UTC 19 Jul 2019 & Minami-daitojima \\
$1-2$ & 0000 UTC 6 Aug 2018 & Minami-torishima & $1-17$ & 1200 UTC 5 Aug 2019 & Kagoshima \\
$1-3$ & 1200 UTC 19 Aug 2018 & Wajima & $1-18$ & 0000 UTC 14 Sep 2019 & Kushiro \\
$1-4$ & 0000 UTC 3 Sep 2018 & Naze & $1-19$ & 1200 UTC 18 Sep 2019 & Kushiro \\
$1-5$ & 1200 UTC 25 Sep 2018 & Sapporo & $1-20$ & 1200 UTC 11 Oct 2019 & Kagoshima \\
$1-6$ & 0000 UTC 28 Feb 2019 & Kagoshima & 1-21 & 0000 UTC 14 Nov 2019 & Kushiro \\
$1-7$ & 0000 UTC 1 Mar 2019 & Hachijojima & 1-22 & 1200 UTC 21 Dec 2019 & Kagoshima \\
$1-8$ & 1200 UTC 11 Apr 2019 & Wajima & 1-23 & 1200 UTC 29 Dec 2019 & Shionomisaki \\
$1-9$ & 0000 UTC 29 May 2019 & Fukuoka & 1-24 & 0000 UTC 3 May 2020 & Shionomisaki \\
$1-10$ & 0000 UTC 31 May 2019 & Akita & 1-25 & 1200 UTC 21 May 2020 & Naze \\
$1-11$ & 0000 UTC 2 Jun 2019 & Matsue & 1-26 & 1200 UTC 25 May 2020 & Wajima \\
$1-12$ & 0000 UTC 14 Jun 2019 & Matsue & 1-27 & 0000 UTC 27 Jun 2020 & Matsue \\
$1-13$ & 1200 UTC 18 Jun 2019 & Fukuoka & 1-28 & 0000 UTC 3 Jul 2020 & Wajima \\
$1-14$ & 0000 UTC 23 Jun 2019 & Minami-daitojima & 1-29 & 0000 UTC 14 Jul 2020 & Matsue \\
$1-15$ & 0000 UTC 5 Jul 2019 & Wajima & & & \\
\hline
\end{tabular}

long as the upper-bound altitudes of the moist layers were consistent with the satellite-estimated cloud-top height. Buoyancy frequencies are calculated from $N_{m}^{2}$ in the assumed saturated layers, whereas $N^{2}$ is used in other subsaturated layers. The definitions of $N^{2}$ and $N_{m}^{2}$ are as follows:

$$
\begin{aligned}
N^{2} & \equiv \frac{g}{\theta} \frac{\partial \theta}{\partial z}, \\
N_{m}^{2} & \equiv g\left[\frac{1+\left(L q_{s} / R T\right)}{1+\left(\epsilon L^{2} q_{s} / c_{p} R T^{2}\right)}\left(\frac{\partial \ln \theta}{\partial z}+\frac{L}{c_{p} T} \frac{\partial q_{s}}{\partial z}\right)-\frac{\partial q_{s}}{\partial z}\right],
\end{aligned}
$$

where $g, \theta, L, q_{s}, R$, and $T$ denote the gravity acceleration, potential temperature, saturation specific humidity, gas constant of dry air, and temperature, respectively. The formula of $N_{m}^{2}$ is slightly simplified from that of Durran and Klemp (1982), ignoring the mixing ratio of condensates.
To illustrate the static stability in moist areas, we use the saturated equivalent potential temperature $\theta_{e}^{*}$ formulated by Bolton (1980) but at a relative humidity of $100 \%$. As $\theta_{e}^{*}$ is conserved through moist pseudoadiabatic processes, the sign of the vertical gradient of $\theta_{e}^{*}$ matches that of $N_{m}^{2}$ in a wet area in the same way that the sign of the vertical gradient of $\theta$ matches that of $N^{2}$ in a dry area.

\section{b. Spectral analyses of satellite images}

As observational evidence for the results of linear stability analyses in section 4, horizontal wavelengths perpendicular to cirrus bands are estimated from satellite images. Previous investigations on the horizontal spacing between cirrus bands (e.g., Ohno and Miura 1982; Lenz et al. 2009) typically yielded wavelengths of approximately 10-20 km. However, small-scale features with wavelengths smaller than a few kilometers could have been (a)

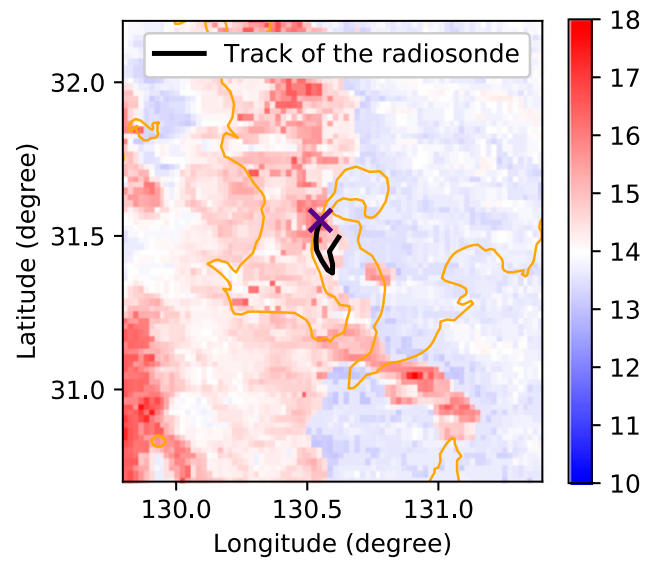

(b) $\begin{array}{lllll}\text { Temperature }\left({ }^{\circ} \mathrm{C}\right) \\ -80 & -60 & -40 & -20 & 0\end{array}$

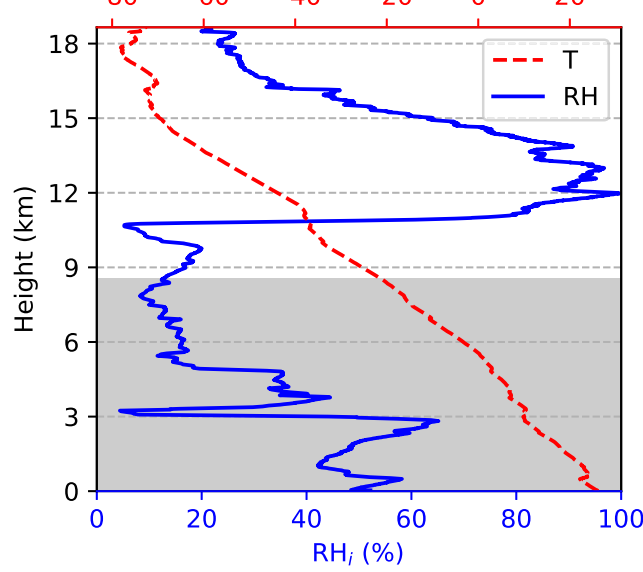

FIG. 1. (a) Satellite-estimated cloud-top height (shading, $\mathrm{km}$ ), horizontal track (black line) and launch site (purple x mark) of the radiosonde, and coastlines (orange line) in case 1-20. (b) Vertical profiles of temperatures (red broken line) and $\mathrm{RH}_{i}$ (blue solid line) observed by the radiosonde in case 1-20. The area below the 350-hPa isobar, which is out of the scope of our cloud detection, is grayed out. 
TABLE 3. Dates, locations, and satellite names of LEO satellite observations used in this study. The rightmost column is the horizontal wavelength $\lambda_{c}$ defined in Eq. (4).

\begin{tabular}{llccc}
\hline \hline Case ID & \multicolumn{1}{c}{ Time and date } & Lon $\left({ }^{\circ} \mathrm{E}\right)$ & Lat $\left({ }^{\circ} \mathrm{N}\right)$ & \multicolumn{1}{c}{ Satellite } \\
\hline 2-1a & 0416 UTC 21 Aug 2018 & 131.3 & 31.0 & \multicolumn{1}{c}{$\lambda_{c}(\mathrm{~km})$} \\
2-1b & 0416 UTC 21 Aug 2018 & 134.8 & 30.6 & Suomi NPP \\
2-2 & 1627 UTC 25 Sep 2018 & 139.0 & 36.9 & NOAA-20 \\
2-3a & 0308 UTC 1 Oct 2018 & 145.1 & 46.9 & Suomi NPP \\
2-3b & 0308 UTC 1 Oct 2018 & 146.1 & 46.0 & Suomi NPP \\
2-4 & 0402 UTC 24 Feb 2019 & 139.9 & 9.5 & Suomi NPP \\
2-5a & 0202 UTC 8 Mar 2019 & 162.5 & 37.5 & Suomi NPP \\
2-5b & 0202 UTC 8 Mar 2019 & 162.3 & 38.0 & Suomi NPP \\
2-5c & 0202 UTC 8 Mar 2019 & 167.2 & 39.2 & Suomi NPP \\
2-6 & 1815 UTC 26 Sep 2019 & 104.6 & -19.6 & Suomi NPP \\
3-1 & 0134 UTC 6 Aug 2019 & 135.6 & 36.3 & Landsat-8 \\
3-2 & 0108 UTC 13 Oct 2019 & 144.0 & 41.9 & Landsat-8 \\
\hline
\end{tabular}

overlooked in those studies because they used relatively lowresolution images from geostationary satellites or infrared channels of the Moderate Resolution Imaging Spectroradiometer (MODIS) instrument, which has a horizontal resolution of $1 \mathrm{~km}$ for thermal infrared bands. To extend their results to smaller horizontal scales, we use two types of high-resolution LEO satellite images: the Visible Infrared Imaging Radiometer Suite (VIIRS) and Landsat-8.

The VIIRS instrument on board LEO satellites Suomi NPP (Cao et al. 2013) and NOAA-20 has 5 high-resolution bands, 16 moderate-resolution bands, and a day/night band. We calculated two-dimensional spectra of brightness temperatures from the I-5 band, which is a high-resolution thermal infrared band with a central wavelength of $11.45 \mu \mathrm{m}$ and spatial resolution of $375 \mathrm{~m}$ at nadir. To avoid the large geometric strain of images off-nadir, cirrus bands more than $500 \mathrm{~km}$ off-nadir are excluded from analyses.

The Landsat-8 satellite (Roy et al. 2014) is a landobserving LEO satellite operated by the U.S. Geological Survey (USGS) and the National Aeronautics and Space Administration (NASA). It has a visible and shortwave infrared imager called the Operational Land Imager (OLI), which has nine spectral bands and features spatial resolutions higher than the VIIRS. In this study, spectral analyses of band 8 were conducted to explore the smaller-scale structures that cannot be resolved by the VIIRS. Band 8 is a panchromatic high-resolution band with an observation wavelength of $0.50-0.68 \mu \mathrm{m}$ and its spatial resolution is $15 \mathrm{~m}$ at nadir.

The target dates and locations of the spectral analyses are shown in Table 3 . These cases are manually extracted from daily global mosaics because of the diverse appearance of cirrus bands. Similar to the case detection for the radiosonde observations, objective detection for target cases, possibly using deep learning techniques presented by Miller et al. (2018), may be a task for future work. Those cases are subjectively sampled from August 2018 to December 2019, rather than being comprehensively collected, due to the lack of an automated and efficient detection algorithm established for infrared images. The cases selected for the spectral analyses are not the same as those of the radiosonde-observed cases, though the sampling periods were chosen to overlap each other, because the location and appearances of cirrus bands often changed substantially within the observation time lag between LEO satellites and radiosondes, which is typically several hours. Observed images are cropped to focus on regions occupied by cirrus bands and their dominant horizontal wavelengths are derived in steps described in the next paragraph. If there are multiple regions that feature cirrus bands with different appearances in a single granule, they are separately cropped and processed as different scenes. For example, in case 2-1 at 0416 UTC 21 August 2018, one region designated as case 2-1a exhibits a horizontally high-wavenumber and highly irregular banding structures, while the other region, named case $2-1 b$, features relatively low-wavenumber and regular waves (Fig. 2).

The procedures for the spectral analyses are as follows: First, the cropped satellite images represented by brightness temperatures are transformed into wavenumber space by the FFT, which is performed in their original pixel coordinates without any regridding to equal-area coordinates to avoid losses of small-scale features arising from interpolations. For simplicity, the horizontal resolution is assumed to be identical to that at nadir everywhere; however, this assumption introduces errors of horizontal scales up to $30 \%$ because the

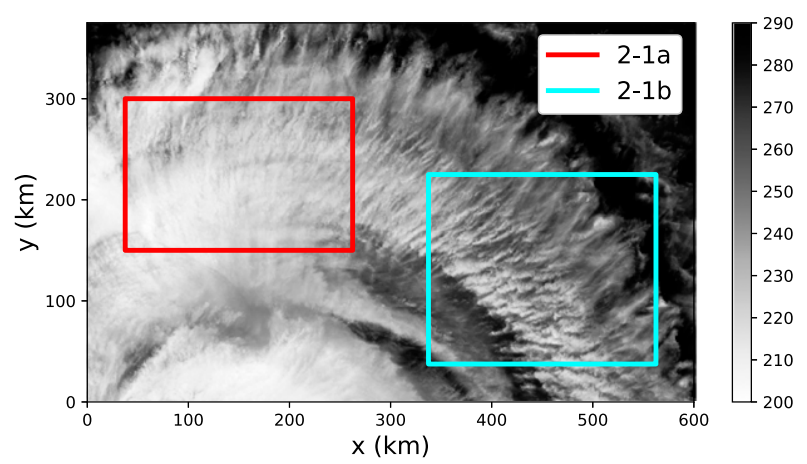

FIG. 2. Infrared satellite brightness temperatures (shading, K) and subregions for cases 2-1a and 2-1b (boxes). 
horizontal resolution is lower off-nadir. For example, the cross-track pixel spacing of the VIIRS I-5 band is approximately $500 \mathrm{~m}$ for pixels $500 \mathrm{~km}$ away from nadir, but is assumed to be $375 \mathrm{~m}$ everywhere. As we aim to obtain a rough estimate of the wavelengths as a reference for section 4, errors of $30 \%$ are acceptable.

Next, the raw spectra are divided by a red noise representing scenes without cirrus bands (figure not shown). The normalized spectra are then transformed from wavelengths in the $x$ and $y$ directions, designated by $\left(\lambda_{x}, \lambda_{y}\right)$, to the horizontal wavelength $\lambda_{H} \equiv\left(\lambda_{x}^{-2}+\lambda_{y}^{-2}\right)^{-1 / 2}$, and the azimuth angle $\theta \equiv$ $\arctan \left(\lambda_{x} / \lambda_{y}\right)$. A two-dimensional Gaussian function defined below is then fitted to the normalized spectra:

$$
f\left(\lambda_{H}, \theta\right)=A \exp \left[-h\left(\lambda_{H}, \theta\right)\right]
$$

where

$$
h\left(\lambda_{H}, \theta\right)=\frac{\left(\log _{10} \lambda_{H}-\log _{10} \lambda_{c}\right)^{2}}{2 \sigma_{\lambda}}+\frac{\left(\theta-\theta_{c}\right)^{2}}{2 \sigma_{\theta}},
$$

where $A, \lambda_{c}, \theta_{c}, \sigma_{\lambda}$, and $\sigma_{\theta}$ are the fitting parameters determined using the least squares method. Finally, $\lambda_{c}$ is designated as the dominant wavelength of the cirrus bands. Results of the Gaussian fitting in three of the analyzed cases are shown in Fig. 3. In case 2-2, for example, the dominant wavelength was estimated to be $7 \mathrm{~km}$ (Fig. 3f, red circle) and its one-sigma interval is 4-10 km (Fig. 3f, red contour). In some cases where prominent bands are aligned nearly parallel to the $x$ or $y$ axis (Figs. 3a,g), the dominant value estimation for either $\lambda_{x}$ or $\lambda_{y}$ was beyond the maximum valid value in the spectrum (Figs. 3b,i). However, the scalar wavelength $\lambda_{c}$ could be determined without troubles because $\lambda_{x}$ and $\lambda_{y}$ are inversely related to $\lambda_{H}$, as defined above.

\section{c. Results}

Figures $4 \mathrm{a}$ and $4 \mathrm{~b}$ show vertical profiles of the relative humidity and $\theta_{e}^{*}$ in case $1-10$. Observational noise is relatively small and thermal structures can be clearly illustrated. In this case, focusing on the upper troposphere above $350 \mathrm{hPa}, \mathrm{RH}_{i}$ is above $80 \%$ in the $8-12 \mathrm{~km}$ MSL layer, which is thus considered saturated. Within the $10-12 \mathrm{~km}$ MSL layer, the vertical gradient of $\theta_{e}^{*}$ is negative, neglecting small-scale fluctuations. Thus, the stratification is estimated to be unstable. Similar unstably stratified layers are identified in cases 1-1 through 1-29 with various thicknesses (Fig. 5a). In the unstable layer of case 1-10, the vertical wind shear predominantly points to the southeast (Fig. 4d), being nearly parallel to the cloud bands extending from the northwest to the southeast (Fig. 4c).

To extract the basic states behind the cirrus bands, rather than raw values likely affected by perturbation fields, we calculate properties of unstable layers, such as thickness, $N_{m}^{2}$, and the absolute values of the angle between the winds and cloud bands seen in satellite images, averaging them for the 29 cases. The averaged properties of the unstable layers are shown in Table 4 and their histograms are shown in Fig. 5. In these unstable layers, the vertical shear vectors are mostly oriented nearly parallel to the observed cirrus bands (Fig. $5 \mathrm{~g}$ ), although there is a caveat that this result contains uncertainties due to the small sample size. In contrast, the angle between the mean wind vector averaged over the unstable layers and the observed bands is more diversely distributed, with some cases near $0^{\circ}$ and more cases near $90^{\circ}$ (Fig. 5h). The unstable stratification and shear-parallel cloud structures are consistent with the theories of thermal-shear instability (Hill 1968; Asai 1970).

While statically unstable layers are found in all cases, the values of dry $N^{2}$ averaged within these unstable layers are mostly positive (Fig. 5d). Thus, it is essential to take the moist effect into account to appropriately diagnose the static stability in cirrus bands. Such an effect has sometimes been neglected due to low temperatures in such areas (Ohno and Miura 1982; Molinari et al. 2014; Duran and Molinari 2016).

The horizontal wavelengths extracted from the VIIRS images are summarized in Table 3 and the spectra for each case are illustrated in Fig. 3 and Figs. A1 and A2 in the appendix. The values of $\lambda_{c}$ are scattered between 2 and $18 \mathrm{~km}$, although some of them may be affected by the geometric strain of the raw pixel coordinates as described earlier, and can be underestimated by up to $30 \%$. Based on this, the dominant wavelengths of the cirrus bands are roughly estimated to be $2-30 \mathrm{~km}$. While the upper portion of these wavelengths is consistent with previous estimates of approximately 10-20 km (Ohno and Miura 1982; Lenz et al. 2009), shorterwavelength cases are included because of the higher resolution of the IR channel of the VIIRS. The swath of Landsat- 8 observation is too narrow to determine $\lambda_{c}$ but no strong signals are found for $\lambda_{H} \leq 1 \mathrm{~km}$ (Fig. A3), which is consistent with the VIIRS spectra. A comparison of these results with the radiosonde observations (Fig. 5a) suggests that the horizontal wavelength is at the same order or one order of magnitude larger than the thickness of the statically unstable layer. That is, the aspect ratios $\lambda / H$ are approximately $1-10$. Similar values have been identified in numerically simulated cirrus bands $(\lambda / H \sim 5-10$; Trier et al. 2010) and horizontal roll vortices observed in the planetary boundary layer ( $\lambda / H \sim 2-10$; LeMone 1973; Weckwerth et al. 1997).

\section{Model experiments}

To investigate detailed inner structures and heat budgets of cirrus bands that cannot be easily derived from radiosonde observations, a TC is simulated in an idealized rotating radiative-convective equilibrium (RCE) framework (Bretherton et al. 2005; Nolan et al. 2007). Here, we focus on cirrus bands around a TC because they appeared more frequently and consistently around TCs than near jet axes in preliminary experiments. The RCE framework is used instead of a simulation of an actual TC because we intend to reproduce cirrus bands in a simple condition that does not suffer from any external interferences from jet streams or nearby disturbances.

\section{a. Model}

We used version 5.2.1 of the Scalable Computing for the Advanced Library and Environment Regional Model (SCALERM; Nishizawa et al. 2015; Sato et al. 2015; Nishizawa et al. 2018), a 3D fully compressible nonhydrostatic model. In all simulations, we used the msternX radiation scheme (Sekiguchi 
Case 2-1b
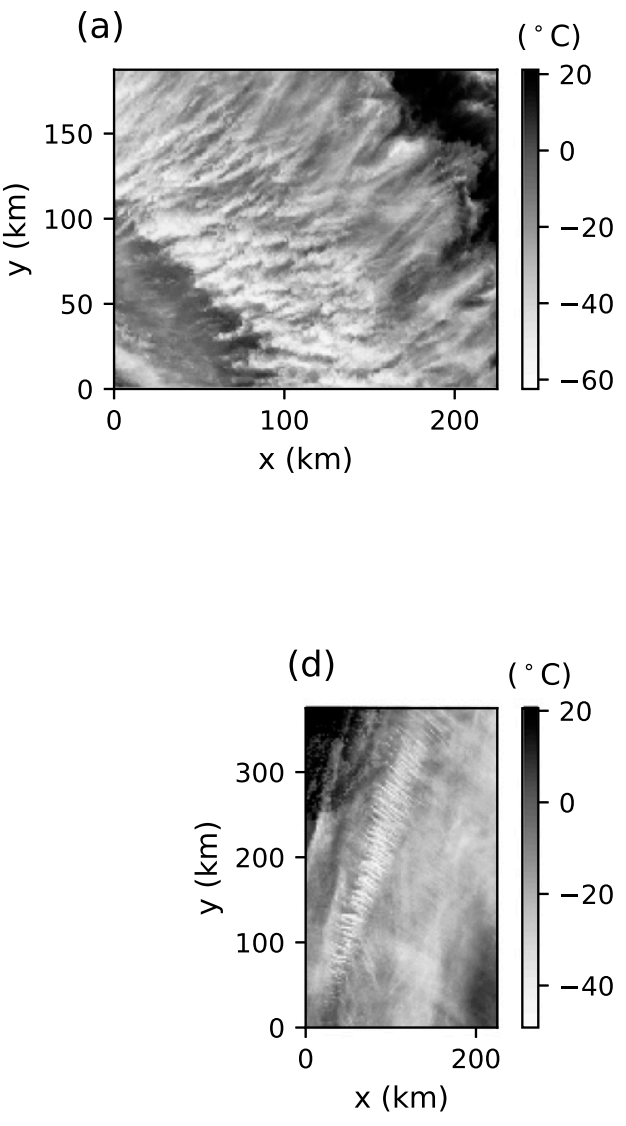

(g)

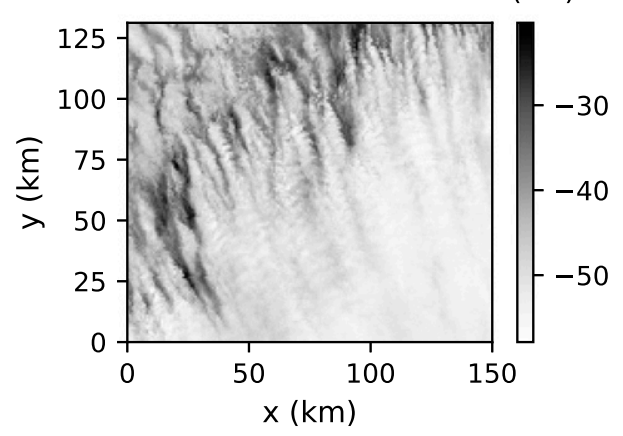

(b)

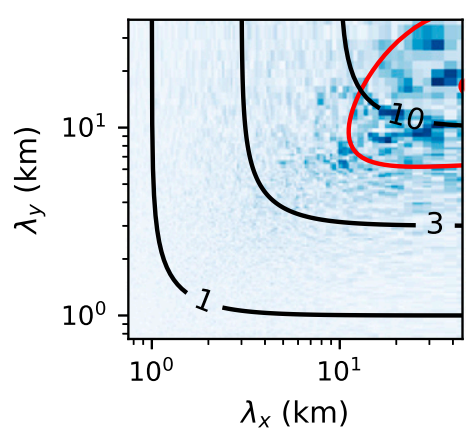

Case 2-2

(e)

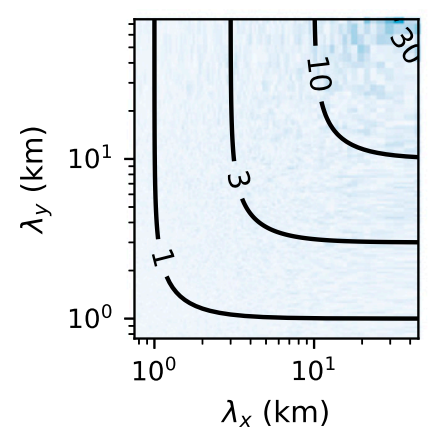

Case 2-5c

(h)

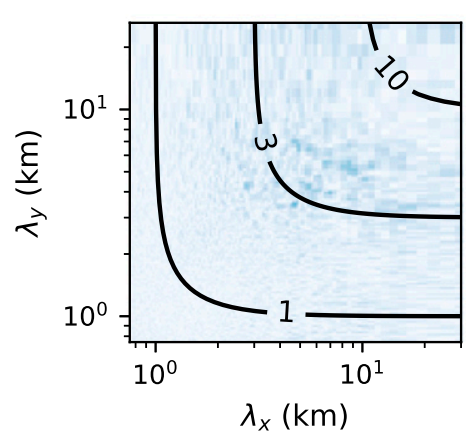

(c)

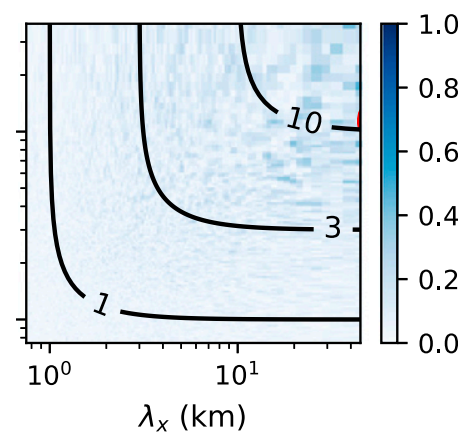

(f)

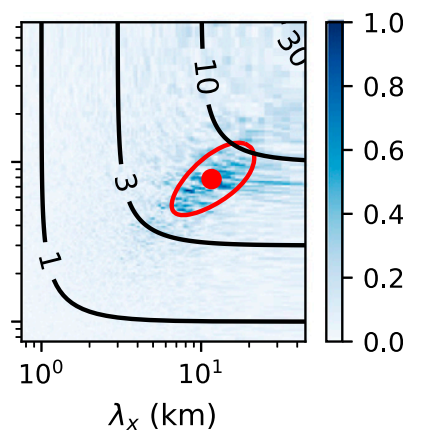

FIG. 3. (a) Satellite infrared brightness temperatures and (b),(c) normalized spectrum of brightness temperatures observed in case 2-1b. The spectrum is divided into two panels: (b) positive $x$-direction wavenumber and (c) negative $x$-direction wavenumber. The black contours in (b) and (c) represent $\lambda_{H}(\mathrm{~km})$. Red dots indicate the center of the fitted Gaussian function and the red contour illustrates its one-sigma interval. (d)-(f) As in (a)-(c), but for case 2-2. (g)-(i) As in (a)-(c), but for case 2-5c.

and Nakajima 2008), a six-class one-moment bulk microphysical parameterization (Tomita 2008), and the MYNN level 2.5 scheme (Mellor and Yamada 1982; Nakanishi and Niino 2004) for planetary boundary layer processes and turbulence.

\section{b. Large-area experiment}

To explicitly simulate cirrus bands spontaneously forming around a quasi-steady TC, a high-resolution simulation in a 
(a)

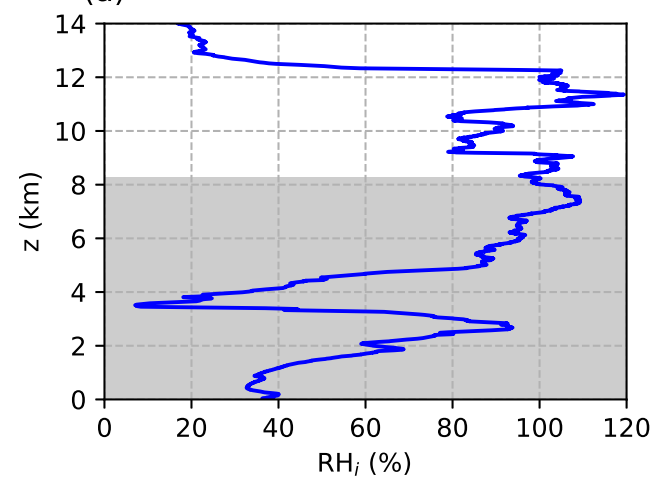

(c)

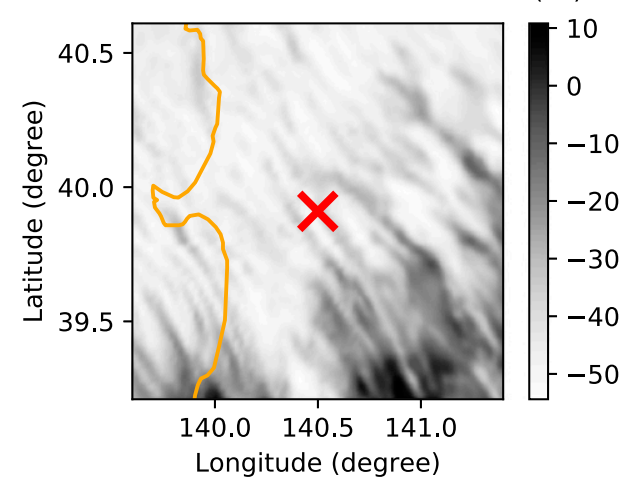

(b)

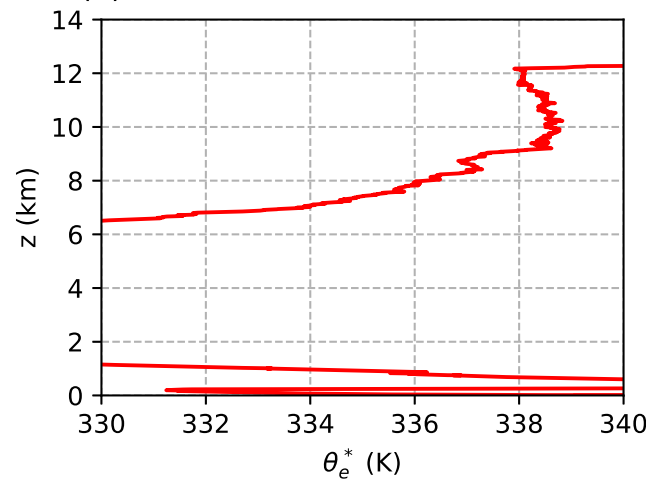

(d)

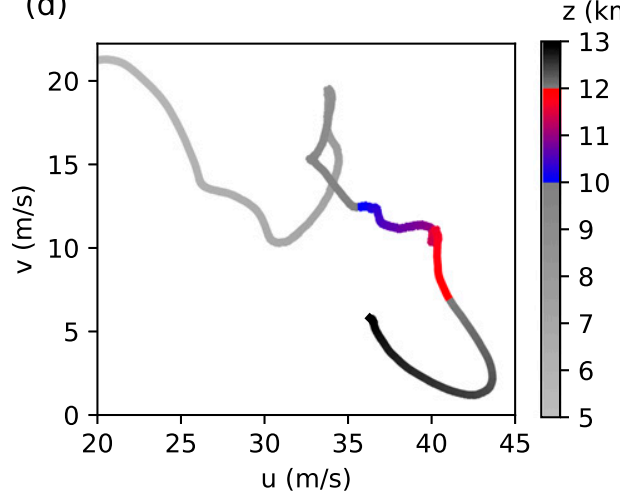

FIG. 4. Vertical profiles of (a) $\mathrm{RH}_{i}$ and (b) saturated equivalent potential temperatures derived from radiosonde observations in case 1-10. (c) Infrared satellite brightness temperature $T_{b}$ (shading, ${ }^{\circ} \mathrm{C}$ ), the location of the radiosonde in case 1-10 at $11 \mathrm{~km}$ MSL (red cross), and coastlines (orange line). (d) Hodograph observed by the radiosonde in case 1-10. The colors of the line denote the altitudes. To focus on the 10-12 km MSL layer, data in the upper and lower altitudes are trimmed and the color shading has discontinuities. In (a), the area below the 350-hPa isobar is grayed out for the same reason as in Fig. 1.

$1280 \mathrm{~km} \times 1280 \mathrm{~km}$ domain was conducted. The Coriolis parameter is set to $1.0 \times 10^{-4} \mathrm{~s}^{-1}$, which is larger than that in the tropics and equivalent to a latitude of $43^{\circ}$, to shrink the TC horizontally (Emanuel 1986) and accommodate the entire radiative-convective equilibrated system in the $1280 \mathrm{~km} \times 1280 \mathrm{~km}$ domain. This experiment is referred to as the large-area experiment in this paper. To reduce computational costs, simulations are conducted in the following three steps:

- initial spinup at a low resolution $(\Delta x=10 \mathrm{~km})$,

- secondary spinup at a moderate resolution $(\Delta x=2 \mathrm{~km})$,

- high-resolution simulation (nested domains with $\Delta x=400 \mathrm{~m}$ in the target region),

where $\Delta x$ denotes the horizontal grid spacing. As summarized in Fig. 6, simulated fields in the initial and secondary spinups are progressively passed to higher-resolution runs.

The configurations that are common in all three steps are described here. Doubly periodic conditions were applied to the lateral boundaries. Sea surface temperatures were fixed at $301.15 \mathrm{~K}$ throughout the region. A time-constant solar flux density of $400 \mathrm{~W} \mathrm{~m}^{-2}$ from the zenith was imposed. Rayleigh damping is deployed in layers above $z=20 \mathrm{~km}$ to reduce the reflection of upward-propagating gravity waves.

\section{1) INITIAL SPINUP}

To obtain an isolated TC in a quasi-steady state, we first perform an initial spinup simulation in a coarse horizontal grid spacing of $10 \mathrm{~km}$. There are 68 vertical levels with the model top at $30.3 \mathrm{~km}$. The vertical grid spacing gradually increases upward. In this step, we deploy a cumulus parameterization (Kain and Fritsch 1989). The initial condition consists of horizontally uniform environmental fields superimposed with an analytical initial vortex, which helps accelerate the TC formation. The environmental fields are computed from temperatures and the relative humidity from the ERA5 (Hersbach et al. 2020) averaged temporally for 1979-2017 in September and spatially for the area of $130^{\circ}-180^{\circ}$ east and $0^{\circ}-20^{\circ}$ north, where tropical cyclones are frequently generated. No environmental winds were applied. The initial vortex is constructed from the formula used by $\mathrm{Xu}$ and Wang (2018) with parameters of $r_{m}=20 \mathrm{~km}, r_{0}=300 \mathrm{~km}, V(0)=20 \mathrm{~m} \mathrm{~s}^{-1}$, and $B=0.7$. Here, $r_{m}, r_{0}, V(0)$, and $B$ denote the radius of maximum wind (RMW), the radius beyond which the tangential wind vanishes, the maximum tangential wind speed near the surface, and a decay 
(a)

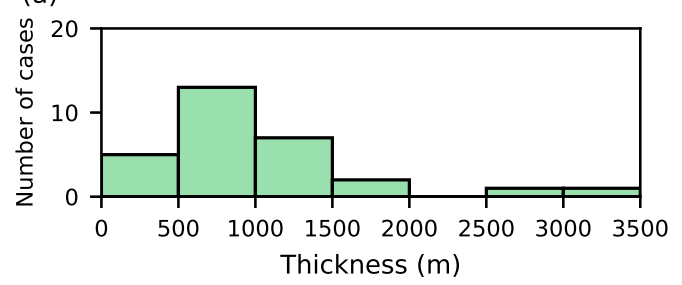

(c)

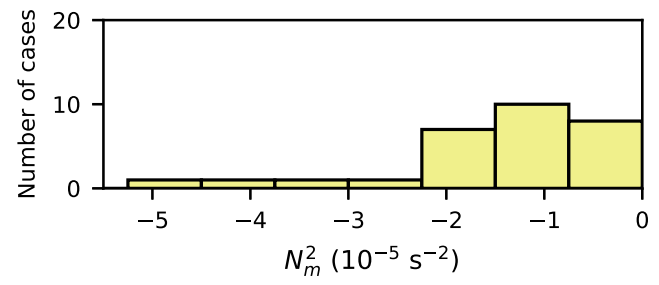

(e)

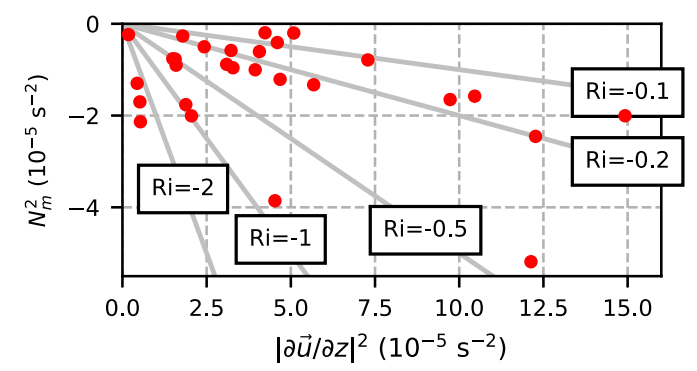

(g)

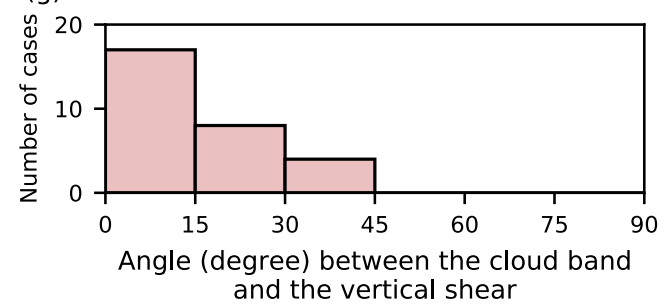

(b)

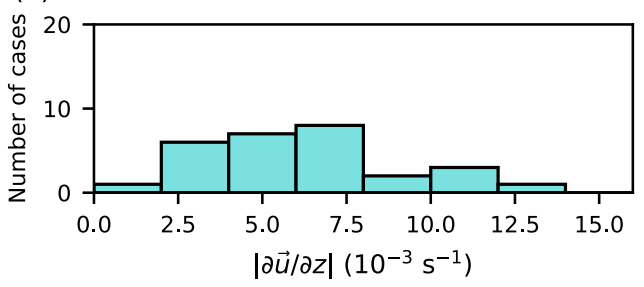

(d)

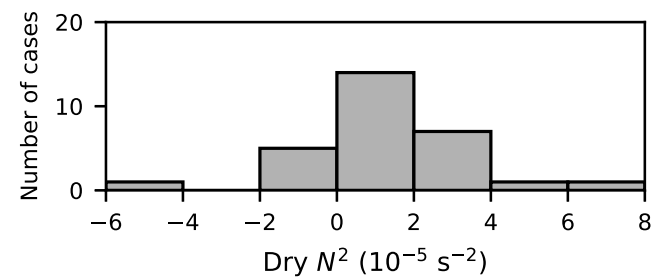

(f)

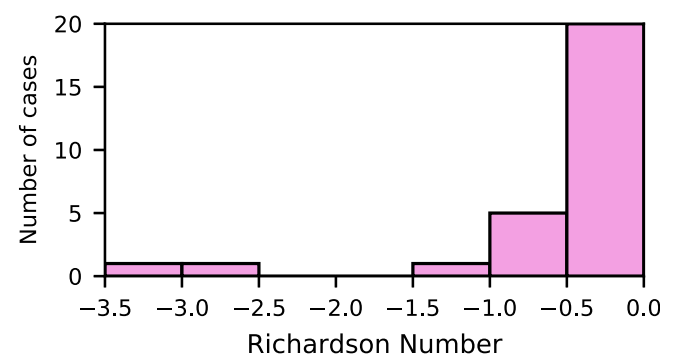

(h)

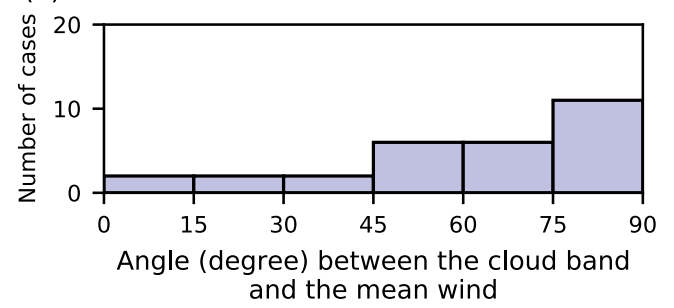

FIG. 5. (a)-(d),(f)-(h) Histograms and (e) a scatterplot of properties of unstable layers found in radiosonde profiles shown in Table 2. The variables shown in each panel are (a) thickness, (b) magnitude of the vertical shear, (c) $N_{m}^{2}$, (d) dry $N^{2}$, (e) $N_{m}^{2}$ and the magnitude of the vertical shear, (f) Richardson number, (g) angle between cloud bands and the vertical shear vector, and (h) angle between cloud bands and the vertical mean wind vector.

factor of the tangential wind with respect to radius outside the RMW, respectively. These vortex fields are added to the environmental fields and integrated for 30 days. The domain-averaged precipitable water became nearly steady by the end of this 30-day run (Fig. 7), suggesting the initial shock was mostly dispersed during this spinup process. Simulation results are temporally and azimuthally averaged over the last three days to obtain radial structures of a nearly steady tropical cyclone, which is used as the initial condition for the second spinup run. The grid points of the minima of the surface pressures were chosen as the origin of the azimuthal averages. The radial averages include the following variables: radial, azimuthal, and vertical wind; temperature; pressure; specific humidity; and the mixing ratio of condensates.

\section{2) SECOND SPINUP}

To obtain structures of a quasi-steady TC at a higher resolution, another spinup simulation was conducted at a moderate resolution. The horizontal grid spacing is $2 \mathrm{~km}$ and there are 210 layers with the top layer at $z=24.9 \mathrm{~km}$, in accordance with the high-resolution run described in the next paragraph. The initial condition is an axisymmetric vortex, the radial structures of which are given from the initial spinup run. Time integration was conducted for three days. The snapshot at the end of this 3day run is used as the initial condition for the subsequent highresolution simulation.

\section{3) High-RESOLUTION SIMULATION}

In this highest-resolution run, cirrus bands on the outflow layers of the TC are explicitly simulated in a one-way nested simulation (Fig. 8). The outer and inner domains have horizontal grid spacings of $2 \mathrm{~km}$ and $400 \mathrm{~m}$, respectively. While the outer domain covers the entire $1280 \mathrm{~km} \times 1280 \mathrm{~km}$ area with doubly periodic conditions, the inner domain has a horizontal extent of $640 \mathrm{~km} \times 640 \mathrm{~km}$ and is centered at the central 
TABLE 4. Properties of statically unstable layers found in radiosonde observations. $N_{m}^{2},|\partial \mathbf{u} / \partial z|$, and Ri are averaged vertically. The angle between cloud bands on satellite images and the vertical wind shear vector is denoted by $\Delta \alpha_{\text {shear. }}$ The angle between the bands and the mean wind vector is denoted by $\Delta \alpha_{\text {mean }}$.

\begin{tabular}{lcc}
\hline \hline \multicolumn{1}{c}{ Variable } & Mean & Median \\
\hline Thickness $(\mathrm{m})$ & 1030 & 730 \\
$N_{m}^{2}\left(\mathrm{~s}^{-2}\right)$ & $-1.4 \times 10^{-5}$ & $-1.0 \times 10^{-5}$ \\
Dry $N^{2}\left(\mathrm{~s}^{-2}\right)$ & $1.0 \times 10^{-5}$ & $0.8 \times 10^{-5}$ \\
$|\partial \mathbf{u} / \partial z|\left(\mathrm{s}^{-1}\right)$ & $7 \times 10^{-3}$ & $6 \times 10^{-3}$ \\
$\mathrm{Ri}$ & -0.7 & -0.3 \\
$\left|\Delta \alpha_{\text {shear }}\right|\left(^{\circ}\right)$ & 14 & 11 \\
$\left|\Delta \alpha_{\text {mean }}\right|\left(^{\circ}\right)$ & 60 & 69 \\
\hline
\end{tabular}

position of the TC in the initial condition. To resolve the inner structures of vertically thin outflow layers of the TC, there are 210 vertical levels with a layer spacing of $100 \mathrm{~m}$ in the upper troposphere. In the inner domain, the uppermost four layers are removed because of a software limitation in the nesting framework. Thus, the model top for the inner domain is at $z=23.4 \mathrm{~km}$, instead of the $z=24.9 \mathrm{~km}$ of the outer domain. This simulation was integrated for $24 \mathrm{~h}$. In this paper, time $t$ is referred to as relative to the start time of this high-resolution run; in other words, the highresolution run is conducted for $t=0-24 \mathrm{~h}$ and only results from $t=12-24 \mathrm{~h}$ are used for analyses. The intensity of the TC was roughly constant throughout the analysis period of $t=12-24 \mathrm{~h}$ : the minimum sea level pressure was around $950 \mathrm{hPa}$ and the maximum $10-\mathrm{m}$ wind speed was about $45-50 \mathrm{~m} \mathrm{~s}^{-1}$.

\section{c. Sensitivity experiments}

Previous studies have investigated the effect of cloudradiation interaction (CRI) on the formation of the cirrus banding by conducting sensitivity experiments without CRIs (Trier et al. 2010; Kim et al. 2014; Trier and Sharman 2016). They found that CRIs contribute to the cirrus band formation to varying degrees, depending on each case. In this study, we reexamine their results by performing heat budget analyses. We conducted two experiments. One is labeled the "control experiment," where the three steps previously described are repeated in the same manner, but in a smaller computation domain and with a larger Coriolis parameter. Owing to the limitations of computational resources, the size of the outer domain is reduced to $640 \mathrm{~km} \times 640 \mathrm{~km}$ and the size of the inner domain is $320 \mathrm{~km} \times 320 \mathrm{~km}$. To accommodate a radiativeconvective equilibrated TC in the small domain, the Coriolis parameter is increased to $2.0 \times 10^{-4} \mathrm{~s}^{-1}$, which is beyond the value which can exist anywhere on Earth. The potential impact of such a large value of $f$ on the results will be discussed in section 4 using linear stability analyses. In a sensitivity experiment named the "NoCRI experiment," we turned off CRIs by configuring the radiative process to ignore any hydrometeor effects. Other settings, such as domain sizes and the Coriolis parameter, are identical to those of the control experiment. The NoCRI experiment was initialized at $t=12 \mathrm{~h}$ with the snapshot of the control experiment at that moment to conduct high-resolution sensitivity experiments with minimal differences in large-scale features; that is, all variables are identical

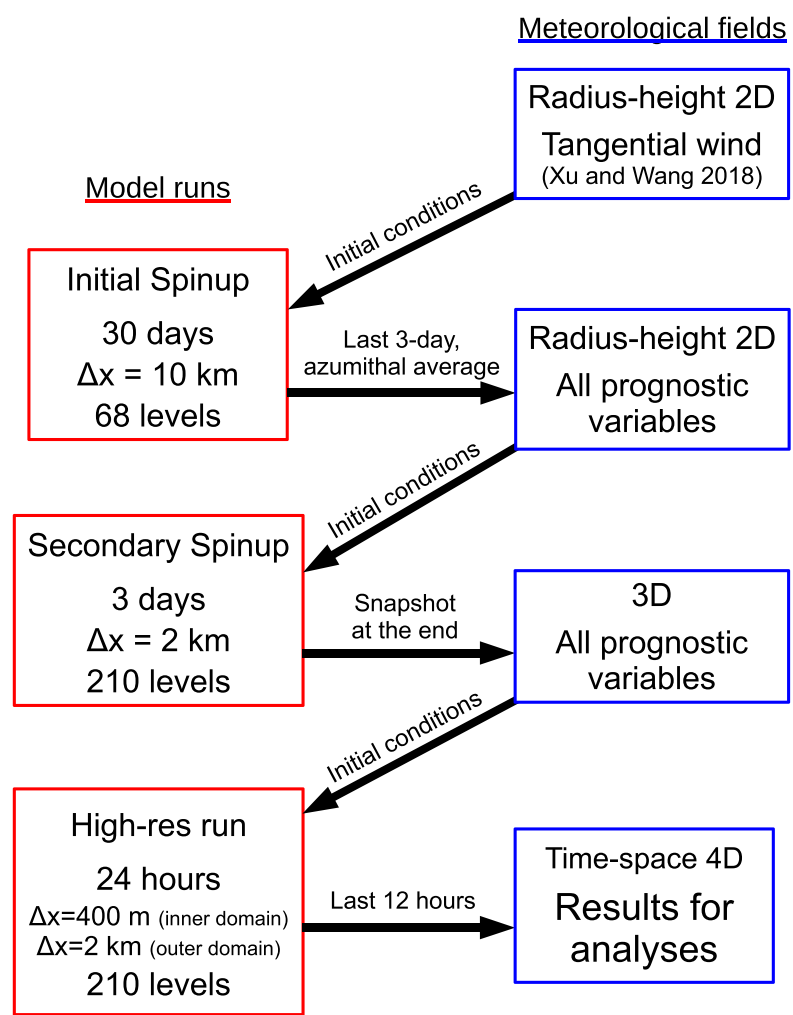

FIG. 6. Outline of the large-area experiment including two spinup runs and a high-resolution run.

at $t=12 \mathrm{~h}$ between the control and NoCRI experiments. We examined the evolution of cirrus bands in the following $12 \mathrm{~h}$. Heat budget analyses were performed in both the control and NoCRI experiments to quantify the effect of CRIs. To illustrate the net heat budget, rather than exchanges between the sensible and latent heat, tendencies are calculated for the moist static temperature (MST), which is defined as the moist static energy divided by the constant-pressure specific heat (Ninomiya 2010; Brown 2011).

\section{d. Results}

\section{1) LARGE-AREA EXPERIMENT}

A snapshot of OLR at $t=22 \mathrm{~h}$ in the large-domain run shows cirrus bands in multiple areas (Fig. 8). To investigate the

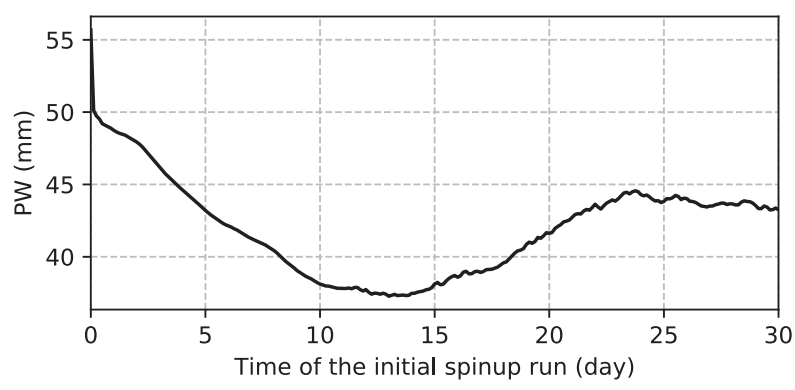

FIG. 7. Time series of the domain-averaged precipitable water in the initial spinup run. 


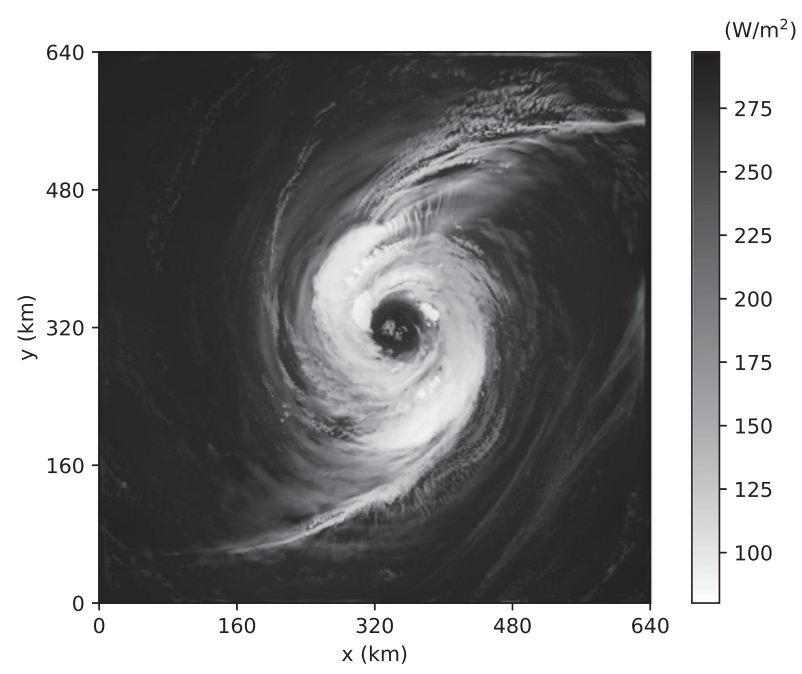

FIG. 8. Snapshot of OLR in the inner domain of the large-area experiment at $t=22 \mathrm{~h}$.

structure of the basic state underlying these bands, the horizontal averages of temperatures, winds, and total hydrometeor mixing ratios were calculated along a line perpendicular to the cirrus bands at $t=21 \mathrm{~h} 10 \mathrm{~min}$ (Fig. 9). The vertical gradient of saturated equivalent potential temperatures $\left(\theta_{e}^{*}\right)$ is negative in the $z=13-14-\mathrm{km}$ layer, where condensates are abundant and thus moist processes are expected to operate, indicating that the moist static stability is negative for $z=13-14 \mathrm{~km}$. In this unstable layer, the vertical wind shear points to the southeast and is nearly parallel to the banding clouds (Figs. 9a,b). These features have also been reported in previous numerical studies (Trier et al. 2010; Kim et al. 2014; Trier and Sharman 2016) and are consistent with the roll-shaped convection driven by thermal-shear instability (Hill 1968; Asai 1970). In addition, these results are consistent with the radiosonde analyses in section 2, which had uncertainties in determining cloudy layers. The evolution of these cirrus bands is investigated by comparing vertical sections between a premature stage $(t=21 \mathrm{~h}$ $10 \mathrm{~min}$; Fig. 10a) and a mature stage ( $t=22 \mathrm{~h} 00 \mathrm{~min}$; Fig. 10b). In the mature stage, the wavy structures of the condensates and vertical winds (Fig. 10d) are stronger than those in the premature stage (Fig. 10c). The horizontal distributions of statically unstable layers are relatively uniform in the premature stage (Fig. 10e) and scattered in the mature stage (Fig. 10f), indicating the perturbation grew intensely enough to considerably distort the background unstable layer.

\section{2) SENSITIVITY EXPERIMENTS}

The evolutions of the OLR in both the control and the NoCRI experiments are shown in Fig. 11. Large-scale features of OLR are almost identical between those experiments. However, no clear cirrus bands were found in the NoCRI experiment, while multiple regions of the cirrus banding can be identified in the control experiment. To quantify this difference, the high-wavenumber components and low-wavenumber components are separated from the OLR fields of each experiment with a $\sigma=1.2-\mathrm{km}$ Gaussian filter. The fields smoothed by this filter are defined as low-wavenumber components, and the residuals are labeled as high-wavenumber components. The resulting low-wavenumber components are highly similar between the two experiments and their correlation coefficients are above 0.9 throughout the simulation period (Fig. 12a). On the other hand, the amplitude of the highwavenumber components in the NoCRI experiment quickly decreases around $t=12.5 \mathrm{~h}$, shortly after the experiment is initiated, and is constantly lower than that in the control experiment (Fig. 12b). This indicates that small-scale features, including cirrus bands, are weakened by turning off the CRI.

To see the effect of the CRI on cirrus bands in detail, snapshots of the vertical sections shown in Figs. 13a and 13b at $t=13 \mathrm{~h} 40 \mathrm{~min}$ are compared. In the control experiment, wavy structures of cloud ice mixing ratios and vertical winds are evident (Fig. 13c) and an unstable layer exists in the upper layer of the anvil (Fig. 13d). These results are similar to those of the large-area experiment. In contrast, hardly any horizontal structures can be found for ice and vertical velocity in the identical vertical section of the NoCRI experiment (Fig. 13c) and the unstable layer on the upper anvil is absent (Fig. 13f). Thus, the CRI helps sustain statically unstable layers and drives the formation of cirrus bands, as shown in previous studies (Trier et al. 2010; Kim et al. 2014; Trier and Sharman 2016). The heat budgets of the control experiment in the same vertical section shown Fig. 13a are shown in Fig. 14b, along with the vertical distribution of cloud ice (Fig. 14a). The radiative process cools the upper half of the clouds $(z=11-12 \mathrm{~km})$, by approximately $0.5 \mathrm{~K} \mathrm{~h}^{-1}$ on average, while heating the lower half $(z=10.2-11 \mathrm{~km})$ at approximately the same magnitude. Thus, radiative processes act to destabilize the cloudy layer and help sustain the roll-shaped convection in the control experiment. In the NoCRI experiment, however, the radiative process weakly cools the entire domain by approximately $0.1 \mathrm{~K} \mathrm{~h}^{-1}$ and has no discernible destabilizing effects on the cloudy layer (Fig. 14e). The results quantitatively confirm that the CRI is responsible for radiative destabilization within the cirrus layer. In addition, gravity waves and differential horizontal advections that occur in mesoscale cloud systems (Trier et al. 2010) and TCs (Duran and Molinari 2019) may also influence static stability and contribute to cirrus band formation in some cases.

Since the incident solar flux in nature varies diurnally, it seems reasonable to assume that the magnitude of the static instability and the frequency of cirrus bands also vary diurnally, probably stronger and more frequent at night. However, such diurnal cycle of the static stability was not detected in observations around TCs (Duran and Molinari 2016). Comprehensive statistics of cirrus bands observed by satellites and further numerical analyses focused on shortwave effects on the static stability will be needed to address this topic.

Finally, for use in stability analyses in the next section, the turbulent dynamic viscosity coefficients $\nu$ and the turbulent Prandtl number (Pr) are computed by averaging them in the same manner as the heat budgets for the control (Fig. 14c) and the NoCRI (Fig. 14f) experiments. The turbulent dynamic 
(a)

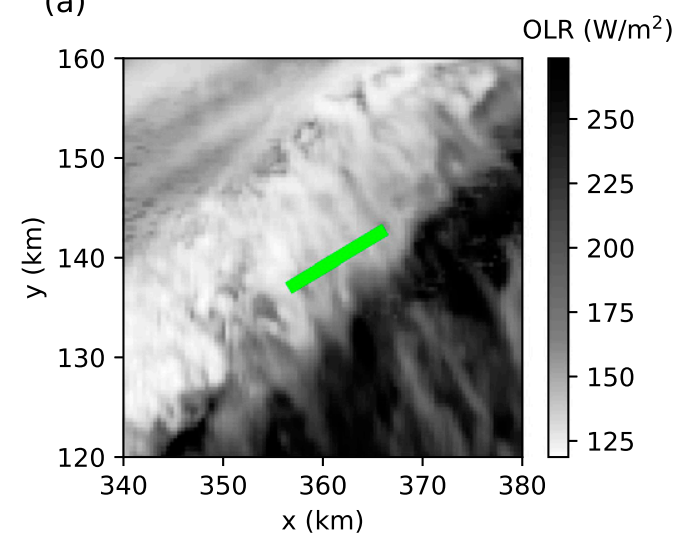

(c)

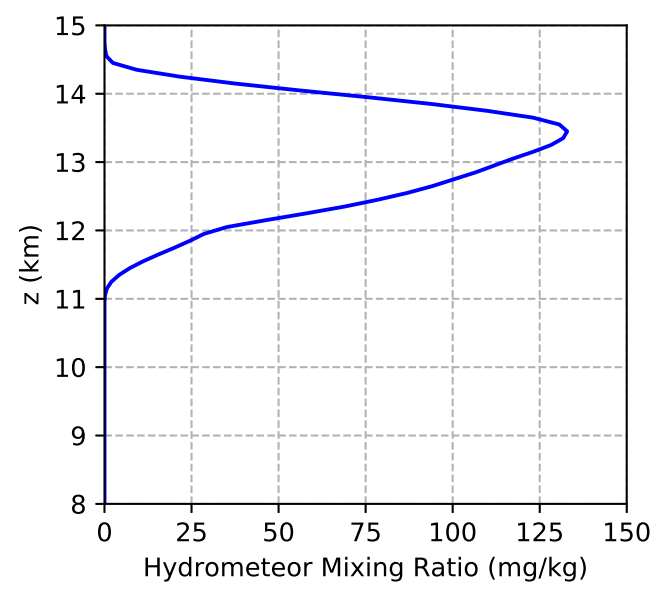

(b)

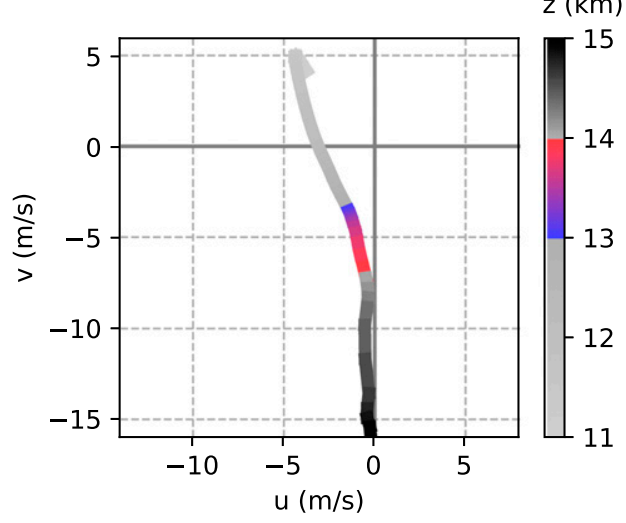

(d)

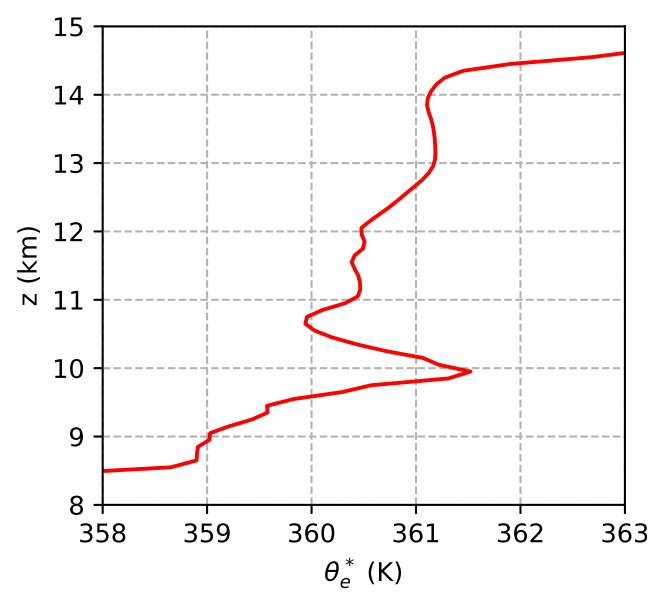

FIG. 9. Snapshots of the large-area experiment at $t=21 \mathrm{~h} 10 \mathrm{~min}$. (a) OLR (shading) zoomed near $(x, y)=$ $(360 \mathrm{~km}, 140 \mathrm{~km})$ and the vertical section (green line) where (b)-(d) horizontal averaging is performed. (b) Hodograph averaged in the vertical section shown in (a). Altitudes are indicated by colors, which are discontinuous to focus on the $z=13-14-\mathrm{km}$ layer. (c) Hydrometeor mixing ratios and (d) saturated equivalent potential temperatures averaged at the same time and vertical section as in (b).

viscosity coefficient varies greatly with height, but is approximately $50 \mathrm{~m}^{2} \mathrm{~s}^{-1}$ in the cloudy layer. The Prandtl number varies between 1 and 4 in the clouds.

\section{Linear stability analyses}

Previous studies have investigated the structures of the thermal-shear instability for constant values of the vertical wind shear and $N^{2}(<0)$ (Hill 1968; Asai 1970), or an unstably stratified Ekman boundary layer (Asai and Nakasuji 1973). These approaches agreed on roll-shaped and shear-parallel structures as the fastest-growing mode. However, their analyses focused on unstable layers with rigid-lid vertical boundaries and the nature of interactions between an unstable layer and neighboring stable layers have not been examined. To overcome these limitations, we perform linear stability analyses with realistic basic states constructed from radiosonde observations and model simulations.

\section{a. Method}

Stability analyses are conducted in a three-dimensional domain $(-\infty<x<\infty,-\infty<y<\infty,-H \leq z \leq H, H \equiv 3 \mathrm{~km})$ governed by the linearized irrotational dry Boussinesq equations:

$$
\begin{aligned}
\frac{\partial u^{\prime}}{\partial t}+\bar{u} \frac{\partial u^{\prime}}{\partial x}+w^{\prime} \frac{\partial \bar{u}}{\partial z} & =-\frac{1}{\rho_{0}} \frac{\partial p^{\prime}}{\partial x}+\nu \nabla^{2} u^{\prime}, \\
\frac{\partial v^{\prime}}{\partial t}+\bar{u} \frac{\partial v^{\prime}}{\partial x} & =-\frac{1}{\rho_{0}} \frac{\partial p^{\prime}}{\partial y}+\nu \nabla^{2} v^{\prime}, \\
\frac{\partial w^{\prime}}{\partial t}+\bar{u} \frac{\partial w^{\prime}}{\partial x} & =-\frac{1}{\rho_{0}} \frac{\partial p^{\prime}}{\partial z}+g \frac{\theta^{\prime}}{\bar{\theta}}+\nu \nabla^{2} w^{\prime}, \\
\frac{\partial \theta^{\prime}}{\partial t}+\bar{u} \frac{\partial \theta^{\prime}}{\partial x}+\frac{\bar{\theta}}{g} N_{e}^{2} w^{\prime} & =\frac{\nu}{\operatorname{Pr}} \nabla^{2} \theta^{\prime}, \\
\frac{\partial u^{\prime}}{\partial x}+\frac{\partial v^{\prime}}{\partial y}+\frac{\partial w^{\prime}}{\partial z} & =0
\end{aligned}
$$



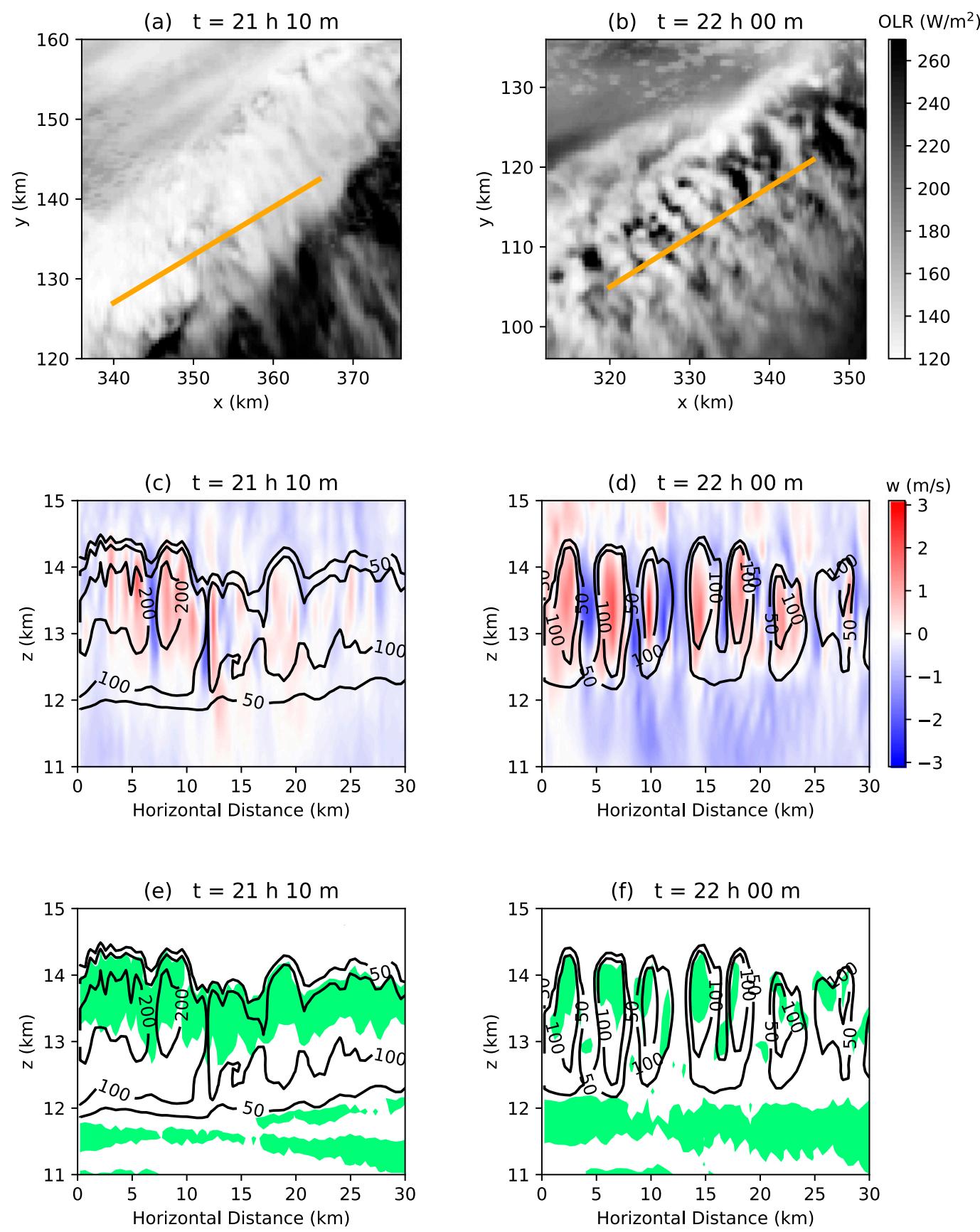

FIG. 10. OLR snapshots at (a) $t=21 \mathrm{~h} 10 \mathrm{~min}$ and (b) $t=22 \mathrm{~h} 00 \mathrm{~min}$, respectively, in the large-area experiment. Orange lines indicate cross sections in other panels. (c),(d) Vertical winds (shading) and hydrometeor mixing ratios (contours, $\mathrm{mg} \mathrm{kg}^{-1}$ ) at (c) $t=21 \mathrm{~h} 10 \mathrm{~min}$ and (d) $t=22 \mathrm{~h} 00 \mathrm{~min}$, respectively, in vertical sections defined in (a) and (b). (e),(f) As in (c) and (d), but green shading for the $N_{m}^{2}<0$ regions.

where $u, v, w, p, \theta, \nu$, and $\operatorname{Pr}$ denote the $x$-, $y$-, and $z$-direction wind velocities, pressure, potential temperature, kinematic viscosity, and Prandtl number, respectively. For any variable $\psi$, overlines $(\bar{\psi})$ and primes $\left(\psi^{\prime}\right)$ represent the basic state and perturbations, respectively. Basic-state winds are assumed to be zero $(\bar{v}=\bar{w}=0)$, except for $\bar{u}$, which will be determined later. As shown in section 2, appropriate treatment of the diabatic processes is important in determining the static stability. In this study, the effect of latent heat release is implicitly expressed through the buoyancy frequency by adopting the "effective buoyancy frequency" $N_{e}^{2}$ defined as follows:

$$
N_{e}^{2}= \begin{cases}N^{2} & \text { for the unsaturated basic state } \\ N_{m}^{2} & \text { for the saturated basic state }\end{cases}
$$


(a)

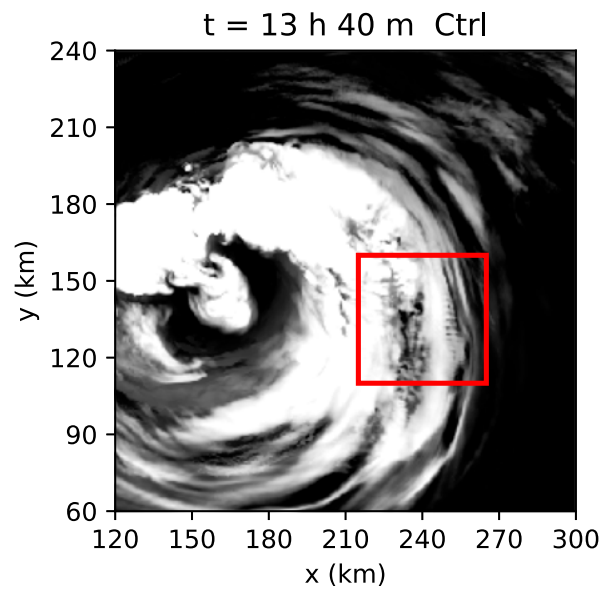

(b)

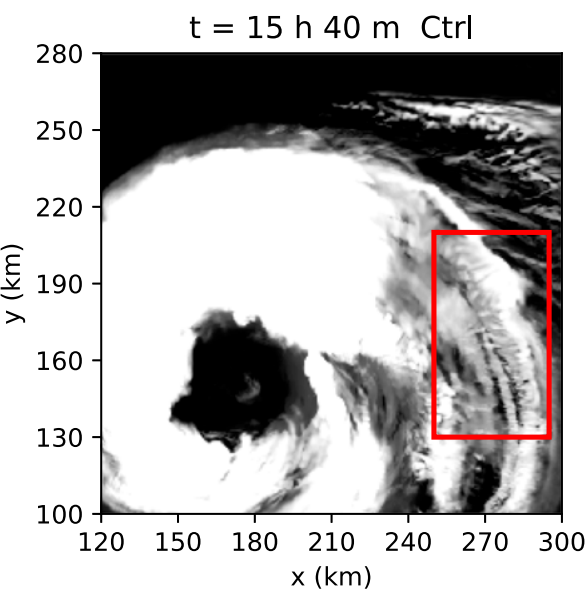

(c)

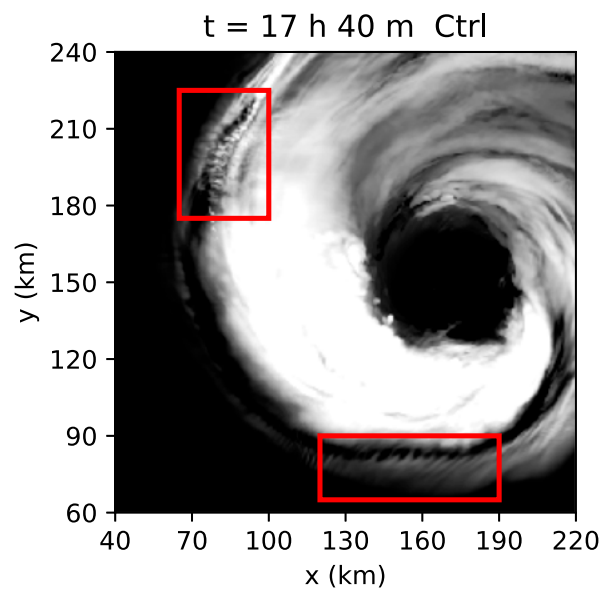

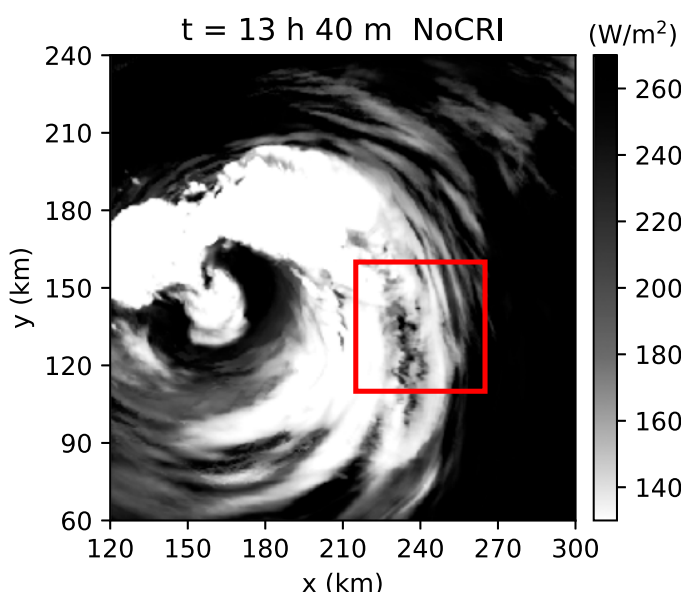
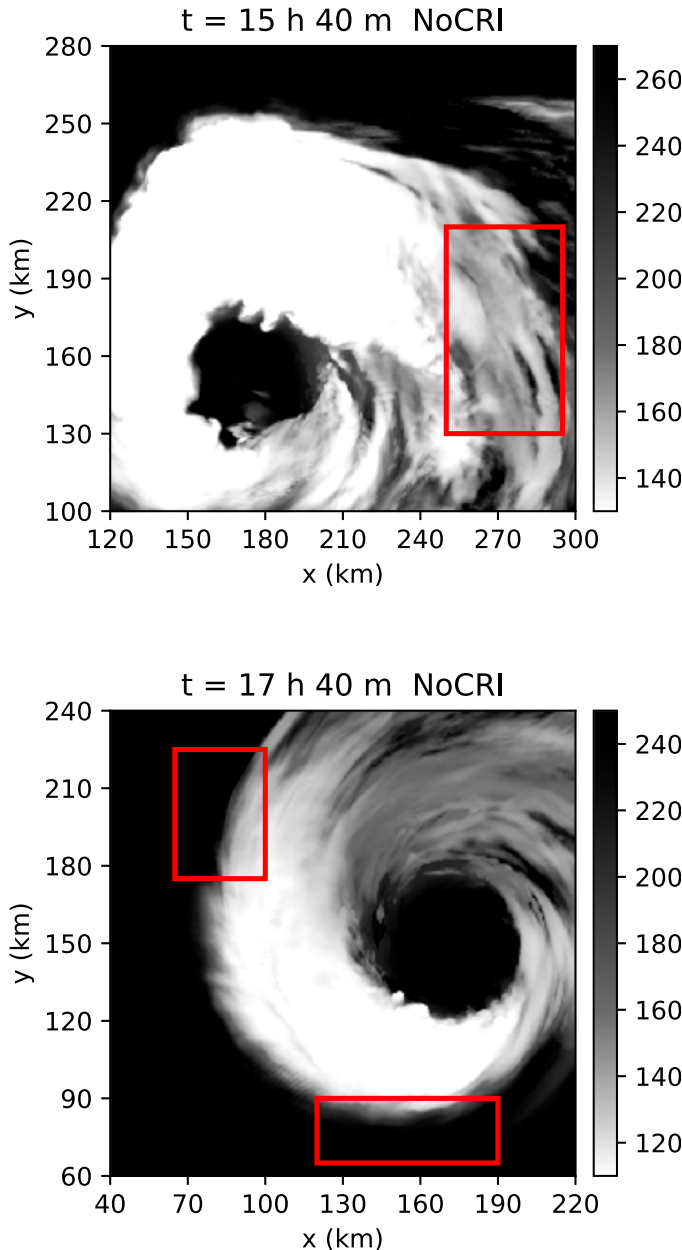

FIG. 11. Snapshots of OLR in the (left) control and (right) NoCRI experiments at (a) $t=13 \mathrm{~h} 40 \mathrm{~min}$, (b) $t=15 \mathrm{~h}$ $40 \mathrm{~min}$ and (c) $t=17 \mathrm{~h} 40 \mathrm{~min}$, respectively. Red rectangles indicate regions featuring cirrus bands in the control experiment. 
(a)

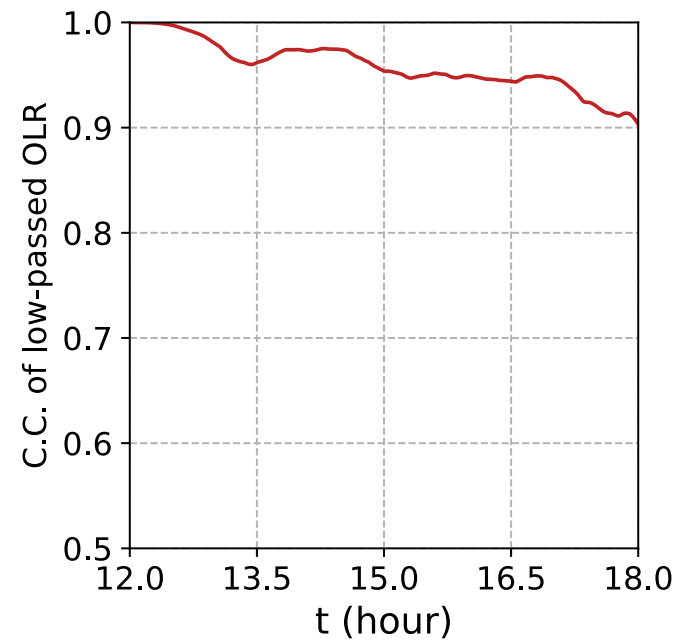

(b)

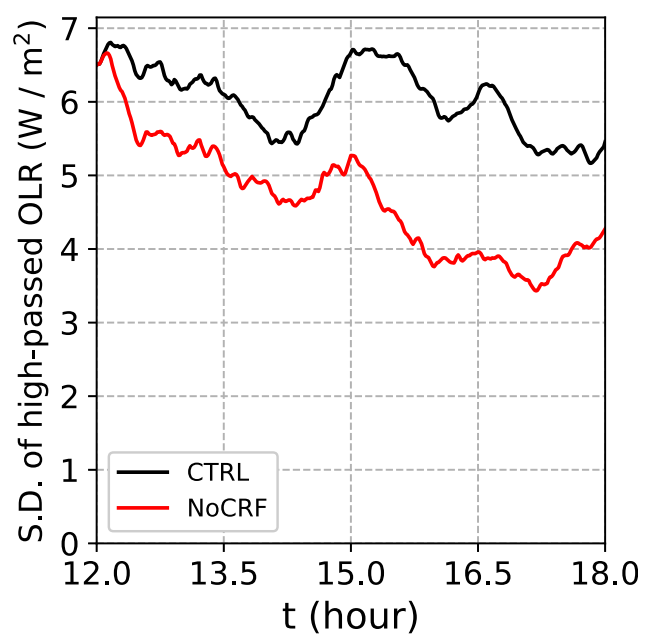

FIG. 12. (a) Correlation coefficients of low-passed OLR between the control and NoCRI experiments. (b) Standard deviations of high-passed OLR for the control experiment (black line) and NoCRI experiment (red line). All values are calculated for the entire inner domain.

Waveform structures are assumed for the horizontal distribution of perturbations at any vertical level and time; that is, any perturbation variable $\psi^{\prime}$ is expressed in the form $\psi^{\prime}(x, y, z, t)=$ $\psi^{\prime}(z, t) \exp (i k x+i l y)$, where $k$ and $l$ denote the wavenumbers in the $x$ and $y$ directions, respectively. After substituting these sinuous structures into Eqs. (5)-(9), the evolution of the field variables becomes

$$
\begin{aligned}
\frac{\partial u^{\prime}}{\partial t} & =-i k \bar{u} u^{\prime}-w^{\prime} \frac{\partial \bar{u}}{\partial z}-\frac{1}{\rho_{0}} \frac{\partial p^{\prime}}{\partial x}+\nu \nabla^{2} u^{\prime} \\
\frac{\partial v^{\prime}}{\partial t} & =-i k \bar{u} v^{\prime}-\frac{1}{\rho_{0}} \frac{\partial p^{\prime}}{\partial y}+\nu \nabla^{2} v^{\prime} \\
\frac{\partial w^{\prime}}{\partial t} & =-i k \bar{u} w^{\prime}-\frac{1}{\rho_{0}} \frac{\partial p^{\prime}}{\partial z}+g \frac{\theta^{\prime}}{\bar{\theta}}+\nu \nabla^{2} w^{\prime} \\
\frac{\partial \theta^{\prime}}{\partial t} & =-i k \bar{u} \theta^{\prime}-\frac{\bar{\theta}}{g} N_{e}^{2} w^{\prime}+\frac{\nu}{\operatorname{Pr}} \nabla^{2} \theta^{\prime} \\
0 & =\frac{\partial u^{\prime}}{\partial x}+\frac{\partial v^{\prime}}{\partial y}+\frac{\partial w^{\prime}}{\partial z}
\end{aligned}
$$

On the bottom $(z=-H)$ and top $(z=H)$ of the domain, we impose the free-slip boundary condition.

Based on the radiosonde observations summarized in Table 4, the basic states are configured as follows:

$$
\begin{aligned}
\bar{u} & =0 \quad \text { at } \quad z=-H, \\
\frac{\partial \bar{u}}{\partial z} & = \begin{cases}u_{z, \text { bottom }}=10 \mathrm{~m} \mathrm{~s}^{-1} \mathrm{~km} & (-H \leq z<-h / 2), \\
u_{z, \text { cloud }}=-5 \mathrm{~m} \mathrm{~s}^{-1} \mathrm{~km} & (-h / 2 \leq z<h / 2), \\
u_{z, \text { top }}=-10 \mathrm{~m} \mathrm{~s}^{-1} \mathrm{~km} & (h / 2 \leq z \leq H),\end{cases} \\
\bar{p} & =200 \mathrm{hPa} \quad \text { at } \quad z=0, \\
\bar{\theta} & =360 \mathrm{~K} \quad \text { at } \quad z=0,
\end{aligned}
$$

$$
\begin{aligned}
N_{e}^{2} & = \begin{cases}N^{2}=10^{-4} \mathrm{~s}^{-2} & (-H \leq z<-h / 2), \\
N_{m}^{2}=-10^{-5} \mathrm{~s}^{-2} & (-h / 2 \leq z<h / 2), \\
N^{2}=10^{-4} \mathrm{~s}^{-2} & (h / 2 \leq z \leq H),\end{cases} \\
\nu & =50 \mathrm{~m}^{2} \mathrm{~s}^{-1}, \\
\operatorname{Pr} & =1,
\end{aligned}
$$

where $h=1 \mathrm{~km}$. The basic state is assumed to be saturated within $-h / 2 \leq z<h / 2$, as implied by the formulation of $N_{e}^{2}$. The values of $\nu$ and $\operatorname{Pr}$ were adopted from the numerical simulations (Fig. 14) because they cannot be directly estimated from radiosonde observations. A value of $\nu=50 \mathrm{~m}^{2} \mathrm{~s}^{-1}$ within clouds appears to be roughly consistent with previous groundbased observations of $10-100 \mathrm{~m}^{2} \mathrm{~s}^{-1}$ (Agee 1975). The wind speed and $\theta_{e}^{*}$ calculated from Eqs. (15) through (20) is illustrated in Fig. 15, along with radiosonde-observed profiles from cases 1-10 and 1-21, in which the properties of the unstable layers are close to the median values shown in Table 4. Because observed profiles are highly diverse, the basic state given by Eqs. (15)-(20) does not closely resemble a specific observed profile, and it was rather designed to capture the mean properties of observed unstable layers shown in Table 4. The nonuniform and asymmetric structure of the vertical wind shear shown in Eq. (16) and Fig. 15d is designed to capture the fact that the vertical shear in the stable layer tends to be stronger than that in the unstable layer in many observed cases (e.g., Fig. 15f).

For these basic states, the evolution of perturbations is calculated using the fourth-order Runge-Kutta method with the following initial conditions:

$$
\begin{gathered}
u^{\prime}=v^{\prime}=w^{\prime}=0, \\
g \frac{\theta^{\prime}}{\bar{\theta}}= \begin{cases}1 \mathrm{~m} \mathrm{~s}^{-2} & (z=0), \\
0 & (z \neq 0) .\end{cases}
\end{gathered}
$$


(a) Ctrl

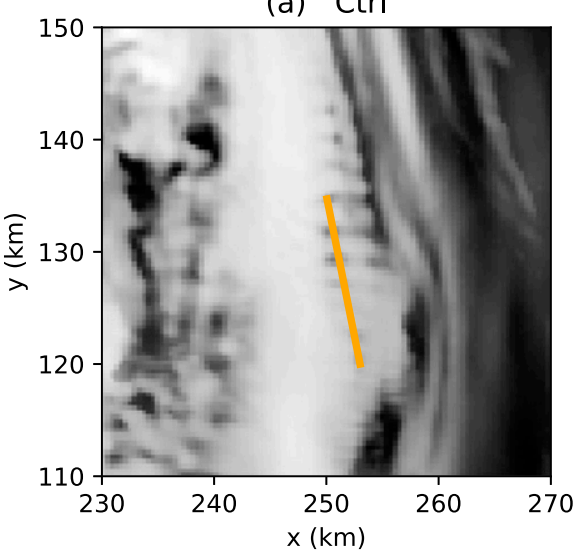

(c) Ctrl

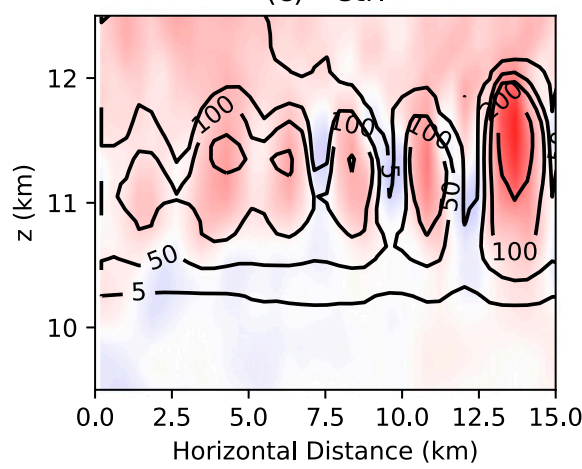

(e) Ctrl

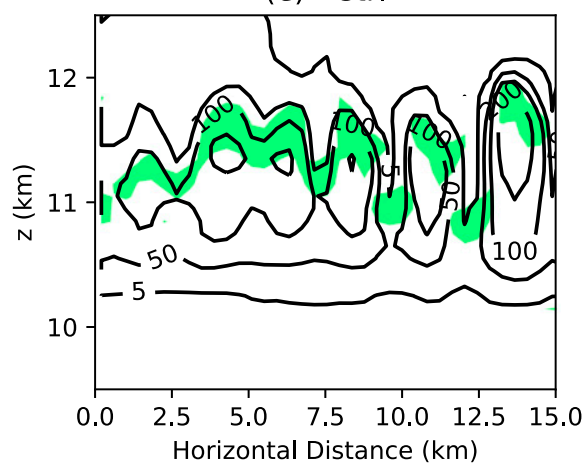

(b) NoCRI

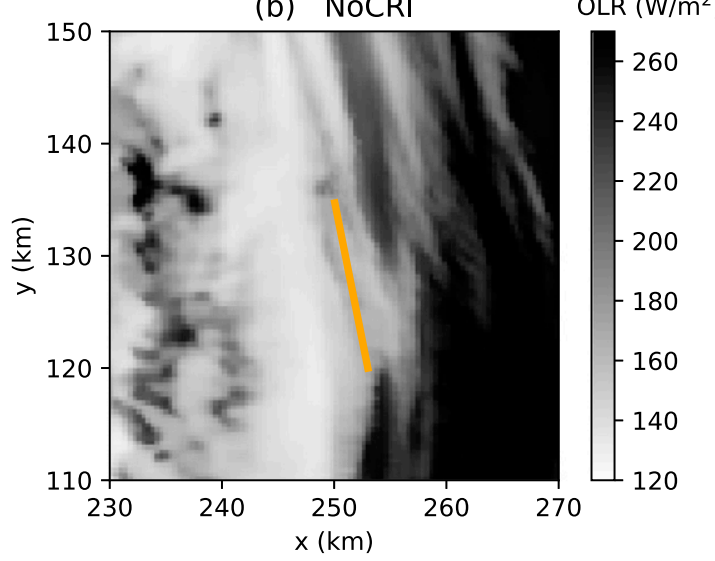

(d) NoCRI

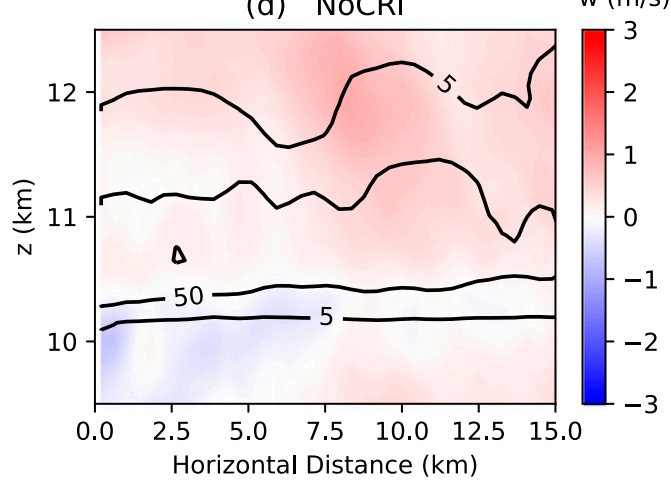

(f) NoCRI

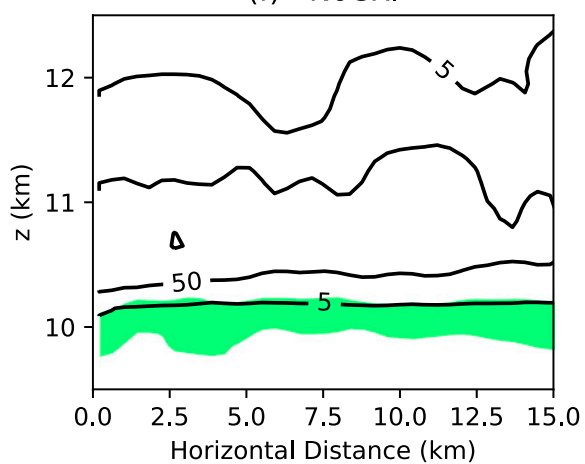

FIG. 13. OLR snapshots at $t=13 \mathrm{~h} 40 \mathrm{~min}$ in (a) the control experiment and (b) the NoCRI experiment, respectively. Orange lines indicate cross sections in the other panels. (c)-(f) As in Fig. 10c-f, but (c),(e) for the control experiment and (d),(f) for the NoCRI experiment, respectively, in vertical sections shown in (a) and (b).

To investigate the impacts of parameter choices in Eqs. (15)(21), sensitivity experiments were also conducted. The detailed configurations of each experiment are summarized in Table 5. In experiment $\mathrm{j}$, a Coriolis parameter of $f=2 \times 10^{-4} \mathrm{~s}^{-1}$ with an $x$-direction gradient of $\bar{\theta}$ satisfying the thermal wind balance is introduced to the basic equations to observe the effect of strong rotation applied in the numerical simulations in section 3 .

\section{b. Results}

Figure 16 illustrates the growth speed $\sigma$, which is defined as the inverse of the $e$-folding time of the perturbation growth, as a function of horizontal wavelengths. In the control experiment (Fig. 16a), the fastest-growing mode is parallel to the vertical shear vector $(k=0)$ and has a growth speed of approximately 
(a)

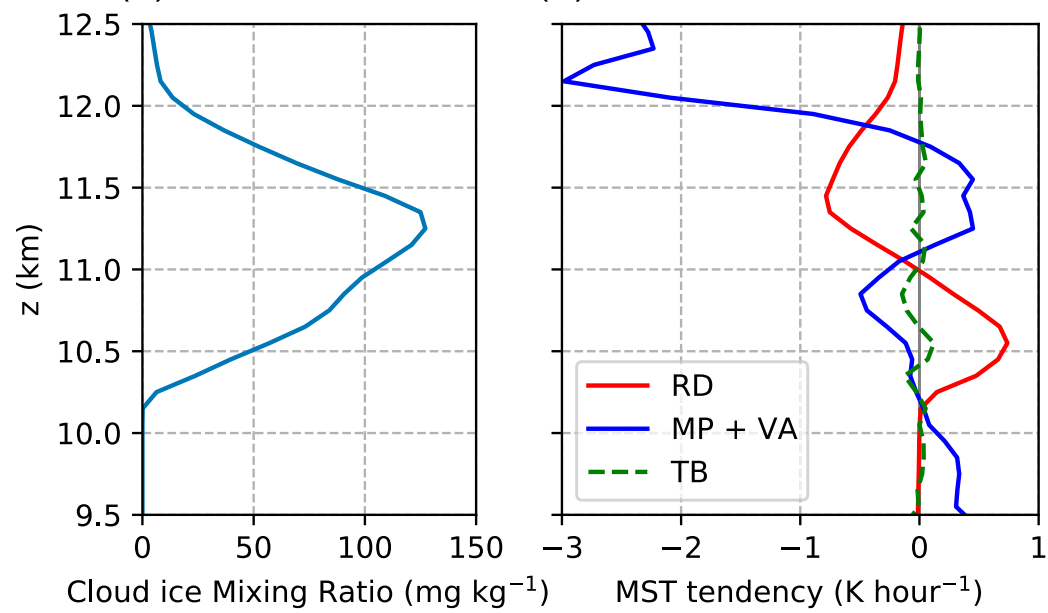

(d)

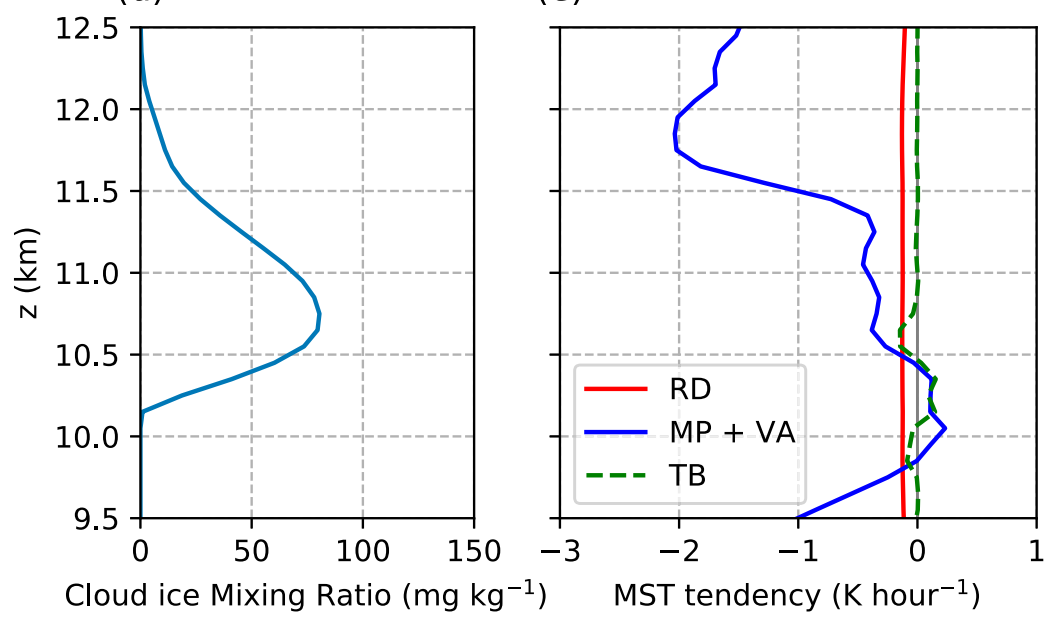

(c)

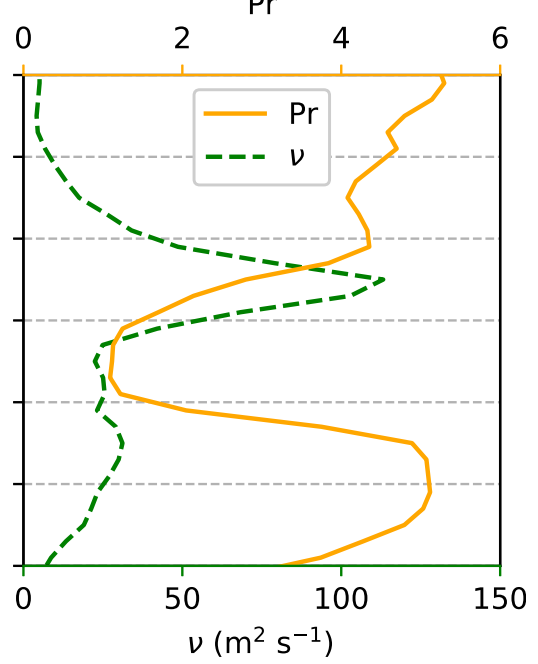

(f)

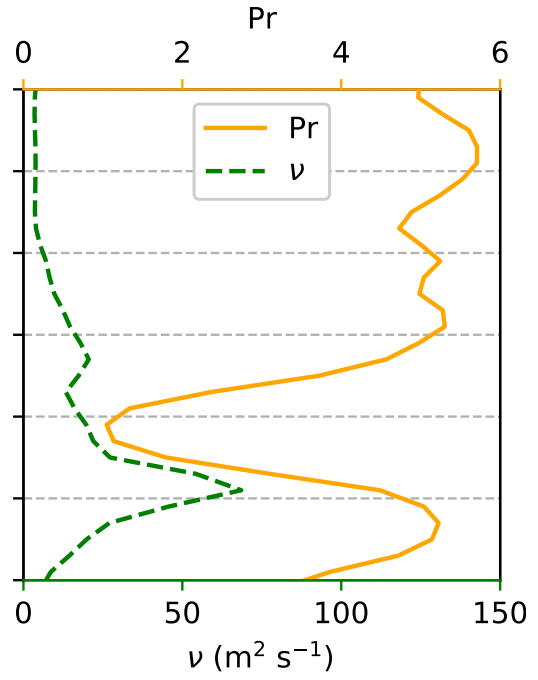

FIG. 14. Horizontal average of (a) cloud ice mixing ratios; (b) tendencies of MST by radiation (RD, red line), microphysics and vertical advection (MP + VA, blue line), and turbulence (TB, green broken line); and (c) Prandtl number (Pr, yellow line) and the kinematic viscosity coefficient ( $\nu$, green broken line), in the cross section shown in Fig. 13a in the control experiment. (d)-(f) As in (a)-(c), but for the NoCRI experiment.

$4 \mathrm{~h}^{-1}$ and a wavelength in the $y$ direction of approximately $2 \mathrm{~km}$. The vertical cross section of this disturbance is roll shaped and localized in the statically unstable layer (Fig. 17a). While the growth speed of this roll-shaped disturbance is not affected by the vertical shear within the statically stable layer (Fig. 16b) or within the unstable layer (Fig. 16c), a value of $N_{m}^{2}$ closer to zero within the unstable layer results in slower growth (Fig. 16d) and a thicker unstable layer allows for faster growth (Fig. 16g). Furthermore, when the unstable layer is replaced with a stable layer, this shearparallel disturbance does not amplify, even if the stratification is as weak as $10^{-7} \mathrm{~s}^{-2}$ (Fig. 16e). These characteristics are consistent with the thermal-shear instability that emerges in a constantly sheared, rigid-lidded unstable layer (Hill 1968; Asai 1970).
The growth speed of the fastest-growing shear-parallel perturbation varies significantly with $N_{m}^{2}$ (Figs. 16d,e), the thickness of the unstable layer (Figs. 16f,g) and diffusive parameters (Figs. 16h,i), but it is almost unaffected by other parameters including the wind shear (Figs. 16b,c,k,1). The wavelength (Fig. 16) and structures (not shown) are similar for all sensitivity experiments, except for the cases with no statically unstable layer (Fig. 16e) or a thinner unstable layer of $h=500 \mathrm{~m}$ (Fig. 16f), in which no shear-parallel modes grew. The fact that the shear-parallel mode did not grow in the $h=500 \mathrm{~m}$ run suggests the critical Rayleigh number $\left(\mathrm{Ra}=\left|N_{m}^{2}\right| h^{4} / \nu \kappa\right)$ lies between 500 (in the $h=500 \mathrm{~m}$ run) and 1000 (in the control run). This is consistent with the simple Rayleigh-Bénard 
(a) Control experiment

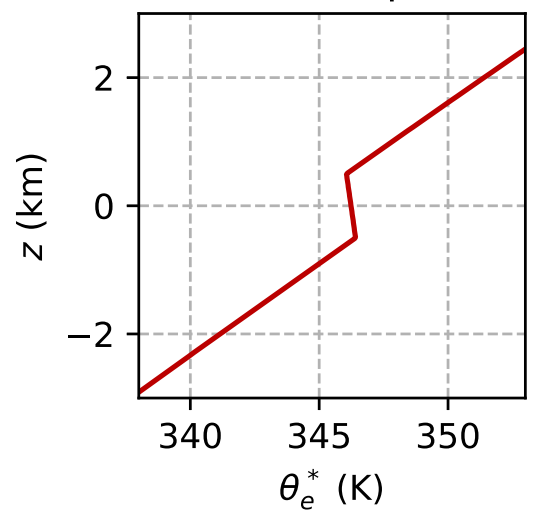

(d) Control experiment

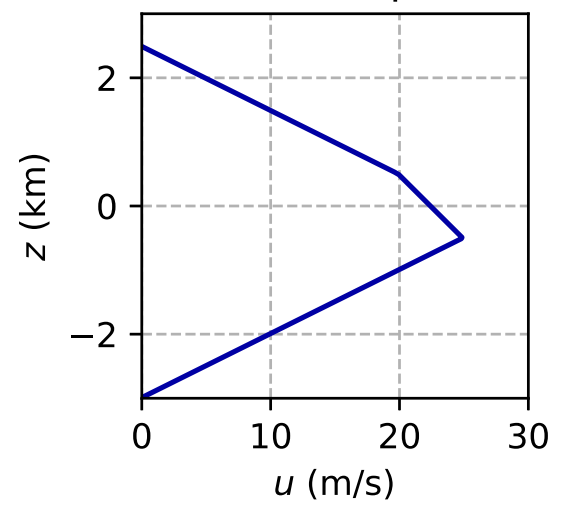

(b)

Case 1-10

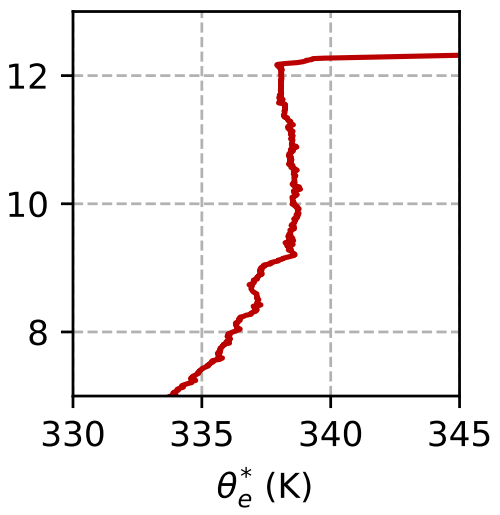

(e) Case 1-10

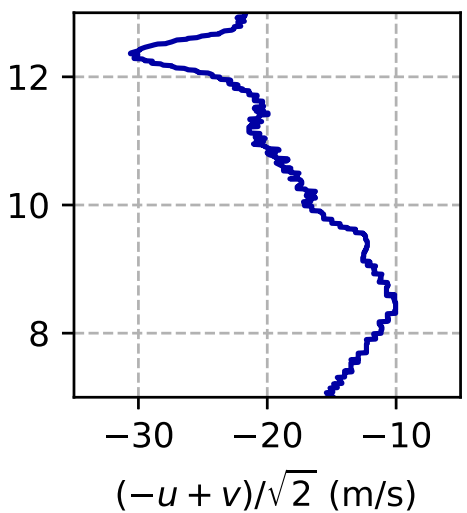

(c) Case 1-21
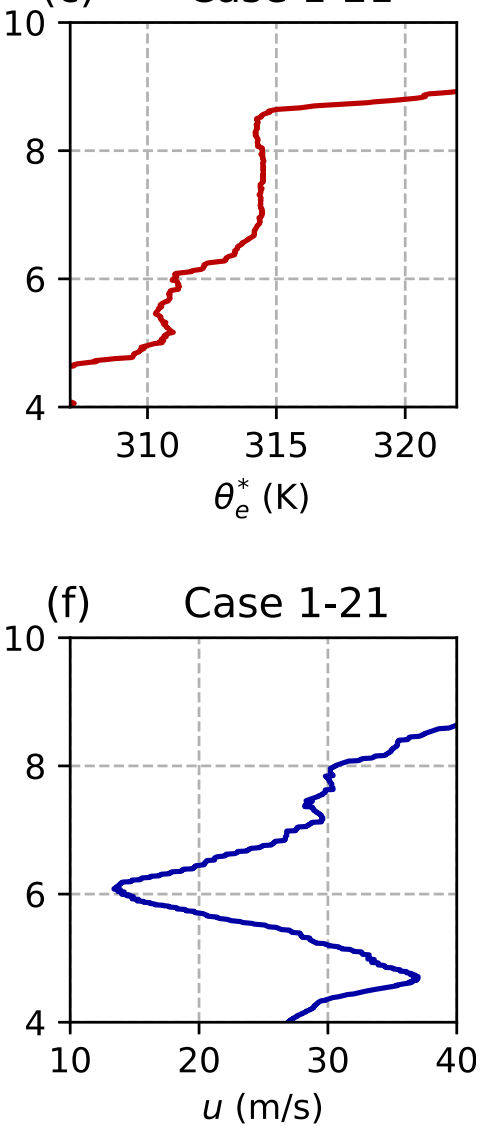

FIG. 15. Vertical profiles of (a)-(c) the saturated equivalent potential temperature and (d)-(f) the wind speed in the (a),(d) control basic state of the stability analysis, (b), (e) case 1-10, and (c),(f) case 1-21. The northwest component of winds are illustrated for case 1-10 because it was the dominant component of the vertical shear vector.

convection with frictionless boundaries, in which the critical Rayleigh number is 657 (Krishnamurti 1968). In reality, the thickness of the unstable layer can be even smaller than $500 \mathrm{~m}$ in some observed cases (Fig. 5a). In cases with thin unstable layers, the small value of $h$ may be compensated by a stronger static instability or weaker turbulent diffusion, or the background unstable layer may be partially hidden by perturbation fields.

Although the growth was slightly enhanced when a large Coriolis parameter was used (Fig. 16j), overall dependence of

TABLE 5. Parameters of basic states in the linear stability analyses. For sensitivity experiments b-1, only values different from the control experiment (a) are shown.

\begin{tabular}{|c|c|c|c|c|c|c|c|c|}
\hline Expt & $u_{z, \text { bottom }}\left(\mathrm{s}^{-1}\right)$ & $u_{z, \text { cloud }}\left(\mathrm{s}^{-1}\right)$ & $u_{z, \text { top }}\left(\mathrm{s}^{-1}\right)$ & $N_{m}^{2}\left(\mathrm{~s}^{-2}\right)$ & $h(\mathrm{~km})$ & Pr & $\nu\left(\mathrm{m}^{2} \mathrm{~s}^{-1}\right)$ & $f\left(\mathrm{~s}^{-1}\right)$ \\
\hline a (control) & $1 \times 10^{-2}$ & $-5 \times 10^{-3}$ & $-1 \times 10^{-2}$ & $-1 \times 10^{-5}$ & 1 & 1 & 50 & 0 \\
\hline $\mathrm{b}$ & 0 & & 0 & & & & & \\
\hline $\mathrm{c}$ & & 0 & & & & & & \\
\hline d & & & & $-5 \times 10^{-6}$ & & & & \\
\hline $\mathrm{e}$ & & & & $1 \times 10^{-7}$ & & & & \\
\hline$f$ & & & & & 0.5 & & & \\
\hline $\mathrm{g}$ & & & & & 2 & & & \\
\hline $\mathrm{h}$ & & & & & & 3 & & \\
\hline $\mathrm{i}$ & & & & & & & 5 & \\
\hline j & & & & & & & & $2 \times 10^{-4}$ \\
\hline $\mathrm{k}$ & $1 \times 10^{-2}$ & $5 \times 10^{-3}$ & $1 \times 10^{-2}$ & & & & & \\
\hline 1 & $1 \times 10^{-2}$ & $-5 \times 10^{-3}$ & $1 \times 10^{-2}$ & & & & & \\
\hline
\end{tabular}


(a)

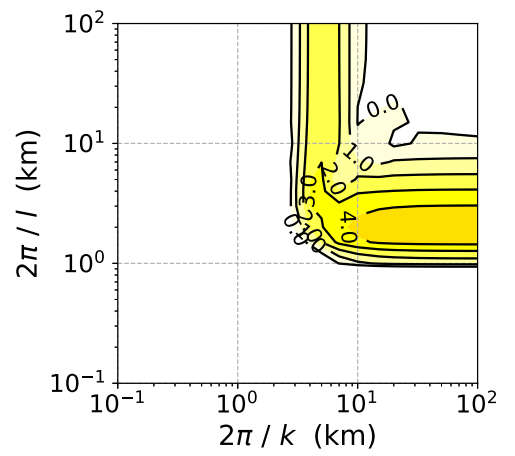

(d)

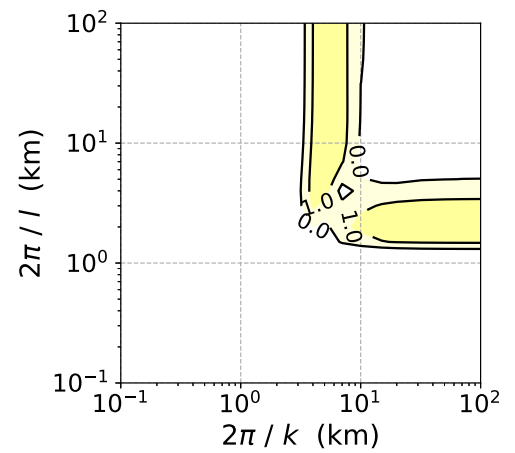

(g)

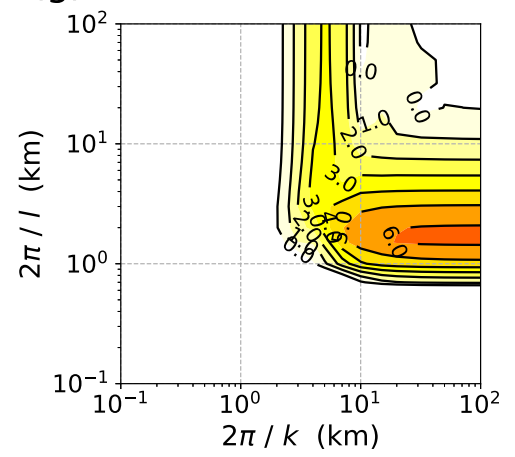

(j)

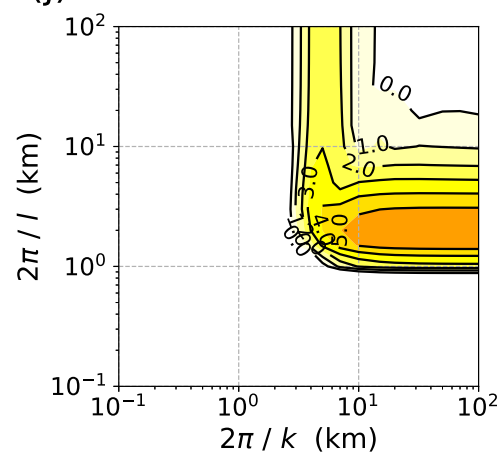

(b)

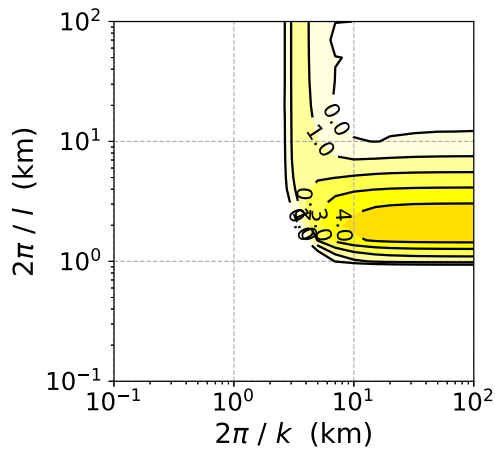

(e)

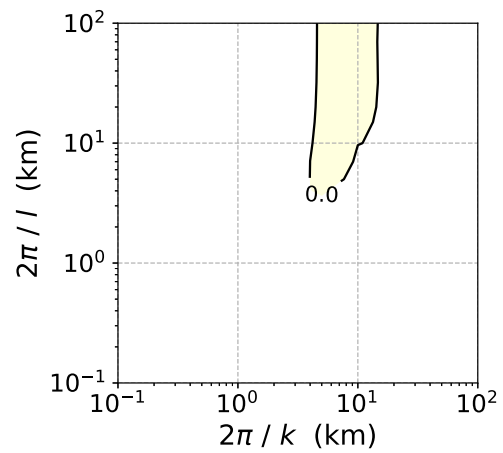

(h)

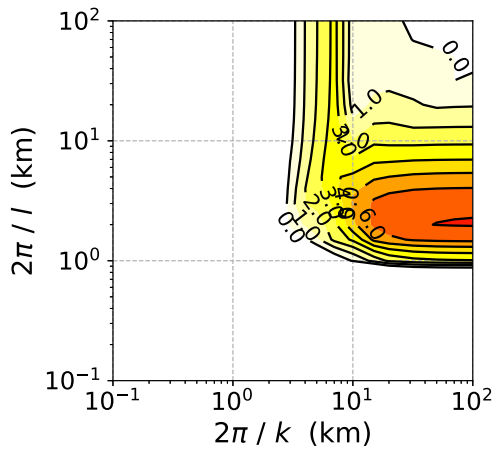

(k)

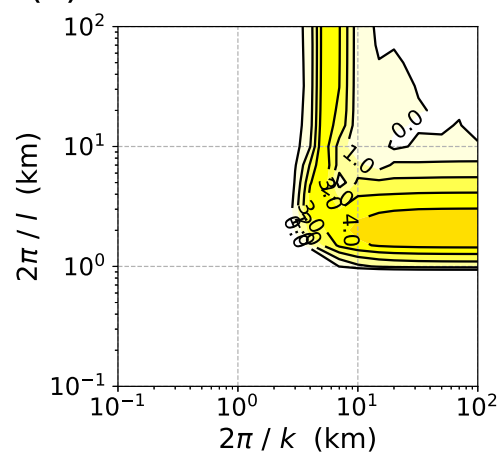

(c)

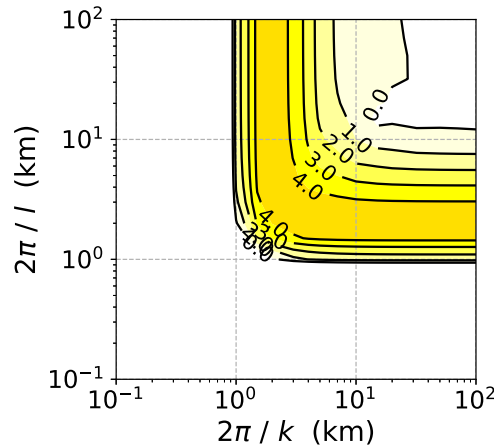

(f)

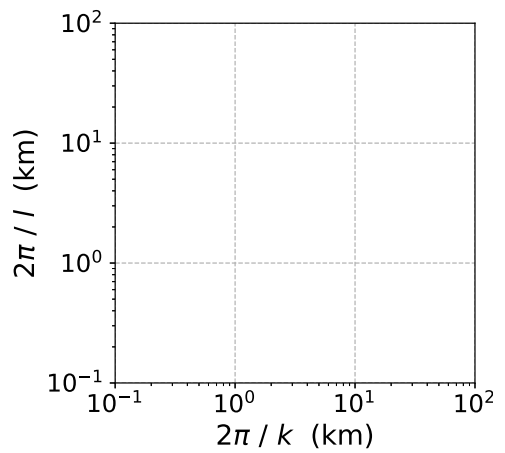

(i)

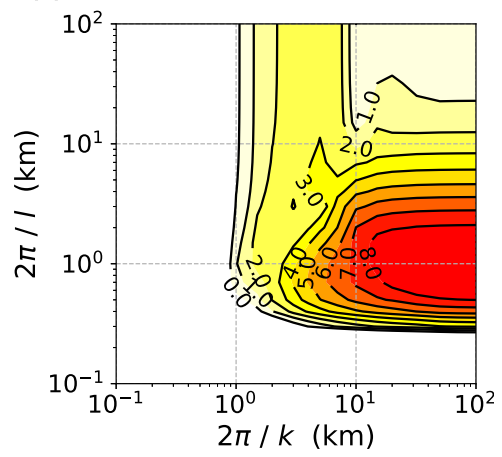

(I)

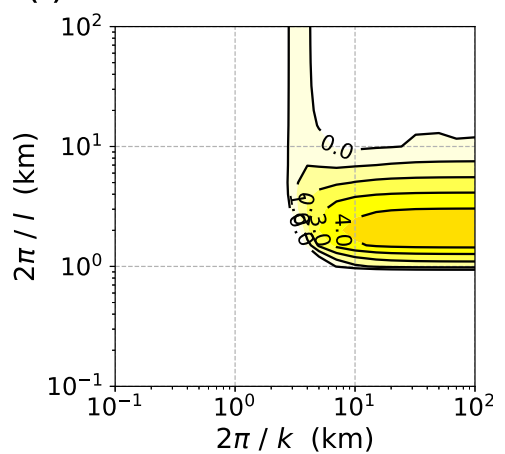

FIG. 16. Growth speeds $\sigma\left(\mathrm{h}^{-1}\right)$ as functions of horizontal wavelengths $(2 \pi / k, 2 \pi / l)$ in basic states described in Table 5. For a clear illustration of the growing modes, contours of growth speeds are only drawn for $\sigma \geq 0$. 
(a)

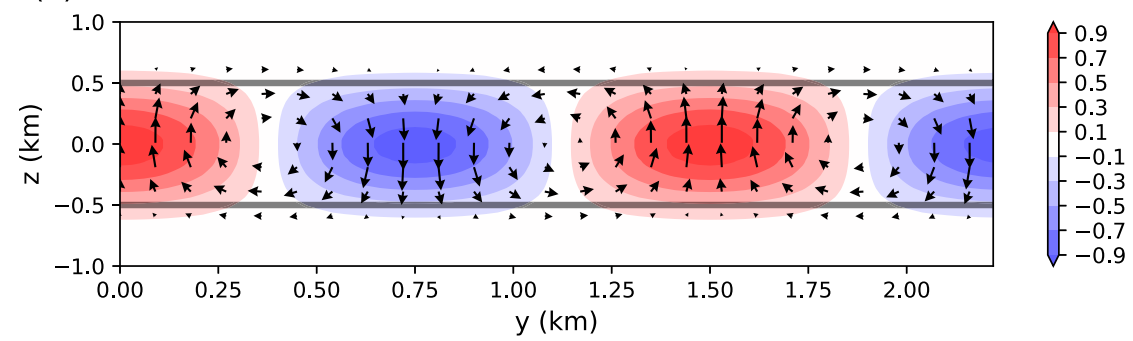

(b)

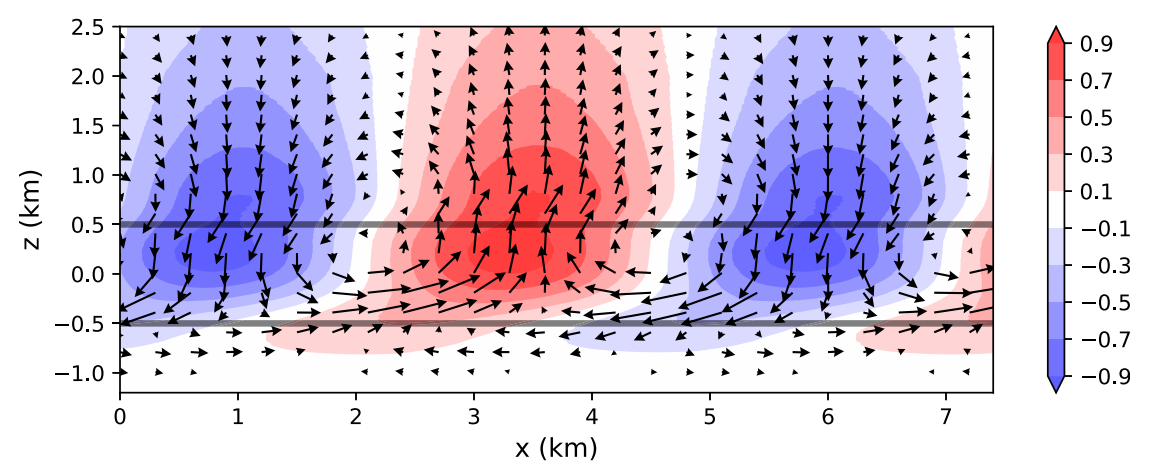

FIG. 17. Vertical velocities (shading) and in-plane winds (vectors) for (a) a shear-parallel mode $(2 \pi / l=1.5 \mathrm{~km})$ in the $y-z$ plane and (b) a cross-shear mode $(2 \pi / k=5 \mathrm{~km})$ in the $x-z$ plane.

the growth speed on wavelengths are highly similar to that in the control experiment. Also, the spatial structure of the shearparallel fastest-growing mode was almost identical to that of the control experiment (not shown). Since the Coriolis parameter in experiment $\mathrm{j}$ is well beyond the realistic range, this result means that the (planetary) rotation is not likely an important factor affecting the thermal-shear instability in actual cirrus bands. This result also suggests that the unrealistically large Coriolis parameters adopted in the numerical simulations in section 3 did not significantly affect the dynamics of the cirrus bands.

The aforementioned shear-parallel disturbance is not the only growing perturbation. Cross-shear disturbances can grow as well, overlapping each other (Fig. 16a). The fastestgrowing mode of cross-shear disturbances $(l=0)$ has an $x$ direction wavelength of approximately $5 \mathrm{~km}$ with a growth speed near $2 \mathrm{~h}^{-1}$. The vertical section (Fig. 17b) indicates that it has a larger vertical scale than the shear-parallel disturbance (Fig. 17a), straddling both the unstable layer and the adjacent stable layer with the same sign of vertical shear as in the unstable layer. This disturbance grows slower when the vertical shear within the stable layer is zero (Fig. 16b). These results indicate that the growth of this deep disturbance depends on the vertical shear in the stable layer, which is similar to the KHI.

In contrast, cross-shear disturbances grow faster when the vertical shear within the unstable layer is weaker (not shown), and even faster when the vertical shear within the unstable layer is zero (Fig. 16c). This is consistent with previous results in the simple Rayleigh-Bénard convection
(Hill 1968; Asai 1970), where vertical wind shear slows down the growth of cross-shear modes.

The growth of this cross-shear mode is strongly enhanced by the unstable stratification of the "cloudy" layer, as the growth slows down to some extent when $N_{m}^{2}$ is closer to zero (Fig. 16e) and is completely suppressed when $N_{m}^{2}$ is barely above zero (Fig. 16f). Compared to the shear-parallel disturbance, this cross-shear disturbance has considerably larger energy transfer from the unstable layer to the neighboring stable layer by pressure perturbations (Fig. 18). This is in part the reason the perturbation in the stable layer can grow exponentially, even though the Richardson number is 1 and neither the thermalshear instability nor the KHI can occur alone (Miles 1961; Howard 1961). The cross-shear mode grows the fastest when there is no change in the sign of the vertical shear (Fig. 16k) and the slowest when the sign changes on both boundaries of the unstable layer (Fig. 161). This also supports the dynamic coupling between statically stable and unstable layers for the crossshear KHI-like mode.

\section{Discussion}

\section{a. Formation mechanism and scale selection of cirrus banding}

Analyses of radiosonde observations in section 2 show that a statically unstable layer likely exists within cirrus bands. Although we assumed moist processes for layers satisfying $\mathrm{RH}_{i}>80 \%$ in the upper troposphere, this assumption may be inaccurate sometimes, and the thickness of 


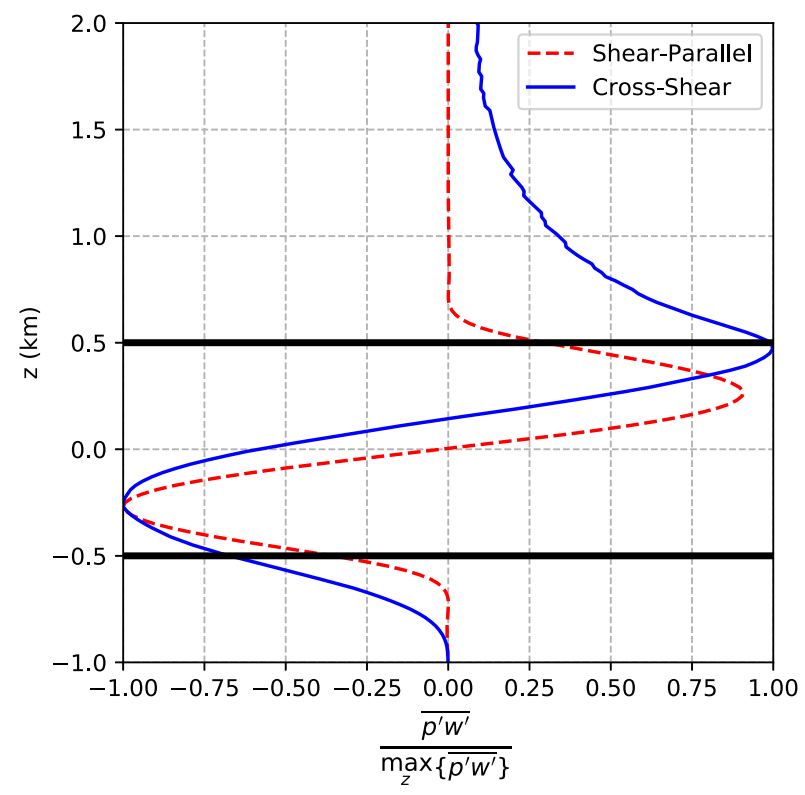

FIG. 18. Horizontally averaged profiles of $p^{\prime} w^{\prime}$ in the shearparallel mode shown in Fig. 17a (red broken line) and the crossshear mode shown in Fig. 17b (blue solid line). Each distribution is normalized by its maximum value in the entire domain.

unstable layers found in the radiosonde analyses includes uncertainties. However, the existence of unstable layers in cirrus bands is supported by the cloud-resolving simulation in section 3 and previous studies (e.g., Trier et al. 2010; Kim et al. 2014; Trier and Sharman 2016). In addition, as in previous works, the orientation of the vertical shear vector within the unstable layer is nearly parallel to the banding clouds. The results from the numerical simulations in section 3 , however, should be treated with some cautions for several reasons. First, the calculation domain is small due to computational limitations, and to accommodate the $\mathrm{TC}$ in this small domain, the Coriolis parameter is unrealistically high especially in the sensitivity experiments. Second, the framework used here is the RCE, in which the environmental profile does not exactly match that in the real tropics.

The linear stability analyses in section 4 demonstrate that the fastest-growing disturbance under a complex basic state constructed from radiosonde observations is also shearparallel roll-shaped convection, suggesting that the simpler theories proposed by Hill (1968) and Asai (1970) also hold for cirrus banding. Thus, at least in the linear regime, it can be concluded that the roll-shaped convection of cirrus banding found in this study is driven by the thermal-shear instability. Our results are consistent with case studies of cirrus bands around a variety of systems, namely, a mesoscale convective system (Trier et al. 2010), an extratropical cyclone (Kim et al. 2014), and a convectively enhanced jet stream (Trier and Sharman 2016). In addition, the nature of the thermal-shear instability is hardly affected by the Coriolis parameter, as noted in section 4 . Therefore, we believe that the limitation of our TC simulations noted above should not be critical for the mechanism of cirrus bands, and the thermal-shear instability is likely an important process in cirrus bands, regardless of their synoptic environment.

One drawback of the linear theory in section 4 is that it cannot explain the upper portion of the wavelength of actual cirrus bands of tens of kilometers. Although the basic states used in our analyses involve uncertainties, the wavelength of the fastest-growing disturbance in the linear regime should not be as large as tens of kilometers, given the insensitivity of the dominant wavelength to various background parameters (Fig. 16). Thus, it seems reasonable to take nonlinear effects into account to further understand the dynamics of cirrus banding. In addition, the possibility of other processes (e.g., gravity waves) contributing to the formation of longwave structures of cirrus bands cannot be ruled out.

Chapman and Proctor (1980) considered a variant of the Rayleigh-Bénard convection, where the heat flux is fixed on the upper and lower boundaries and the Rayleigh number is slightly larger than the critical Rayleigh number. It was found that the horizontal wavelength of a growing disturbance expands indefinitely with time in the nonlinear regime. Although this framework lacks background vertical shear and thus cannot be directly applied to cirrus bands, the constant-heat-flux boundary condition seems to have some relevance to cirrus bands, as they appear to be maintained by a finite amount of radiative heat flux, rather than the constant-temperature condition used in idealized theories (Hill 1968; Asai 1970). In addition, the Rayleigh number in mature cirrus bands is likely to be effectively small, as unstable and stable areas are mixed in such bands in the numerical experiments (Fig. 10e). Thus, nonlinear effects similar to those discussed by Chapman and Proctor (1980) may extend the horizontal wavelengths of cirrus bands. While these nonlinear dynamics can potentially explain a discrepancy between the linear fastest-growing mode and observed cirrus bands, the quantitative verification of these effects remains an interesting topic for future studies.

\section{b. KHI-like disturbances and its possible relation to "fishbone-shaped" cirrus bands}

In section 4, we identified a cross-shear, KHI-like disturbance that grows slowly, straddling both statically stable and unstable layers. As it has a cross-shear wavy structure and a smaller growth speed, it is unlikely to be dominant in actual cirrus bands, which have nearly shear-parallel structures (Fig. 5g). However, some cases manifest fishbone-like structures with cross-band waves (Fig. 3g) and might be attributed to the KHI-like cross-shear instability. These fishbone-shaped cloud bands may be of the same nature as the KHI billows investigated by Trier and Sharman (2016), while the intensity of unstable stratification can be different from that shown by Trier and Sharman (2016). This topic deserves further observational and numerical investigations in the future.

\section{c. Possible effect on TC intensities}

The intensity of TCs is affected by many factors, such as SSTs (DeMaria and Kaplan 1994), subsurface temperatures in 
Case $2-1 a$

(a)

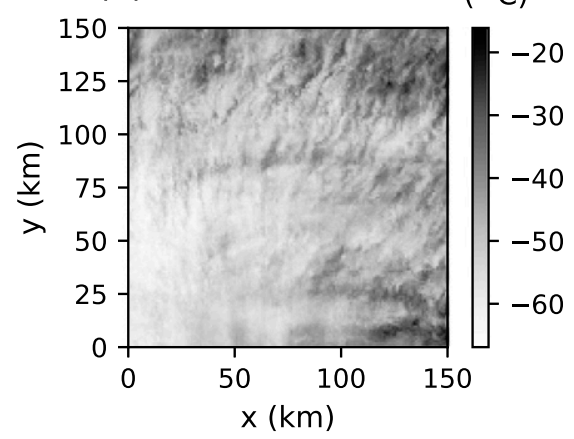

(d)

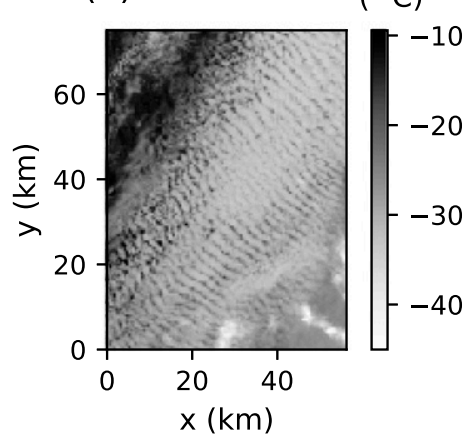

(g)

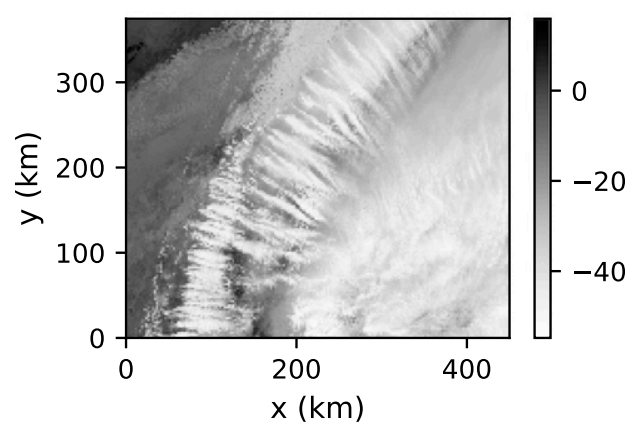

(b)

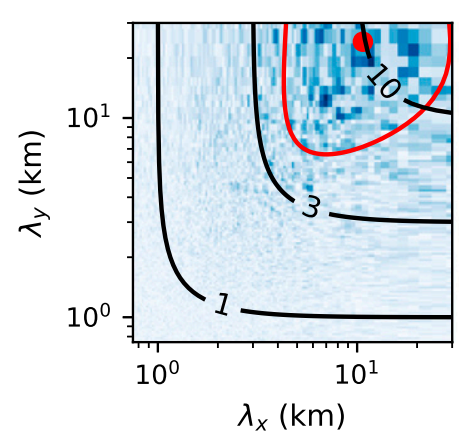

Case 2-3a

(e)

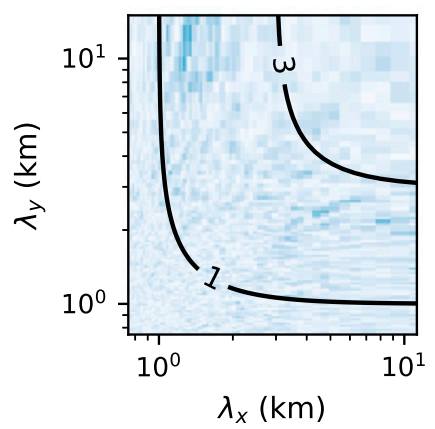

Case $2-3 b$

(h)

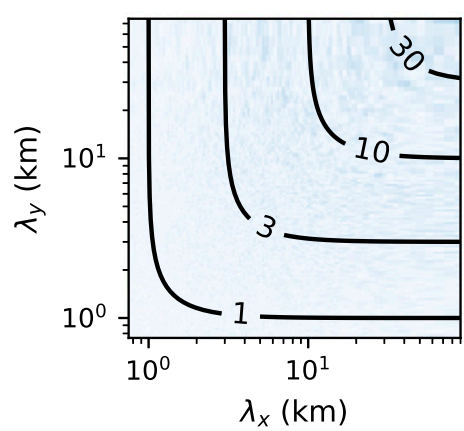

(c)

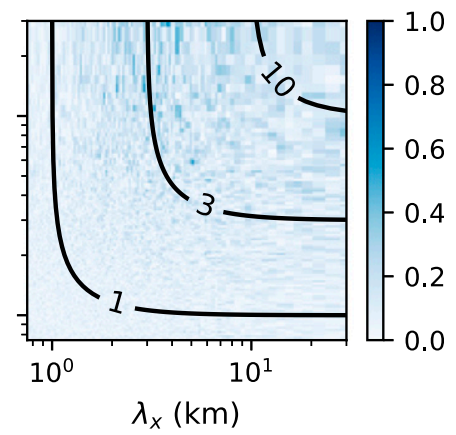

(f)

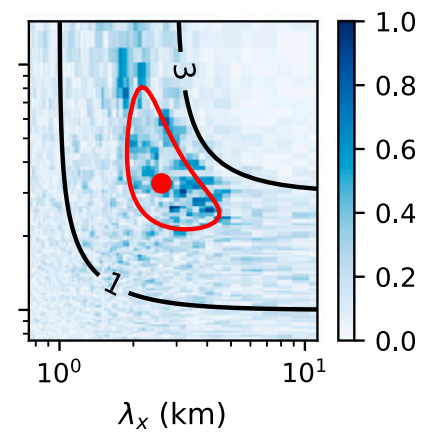

FIG. A1. As in Fig. 3, but for (a)-(c) case 2-1a, (d)-(f) case 2-3a, and (g)-(i) case 2-3b.

the upper ocean (Wada and Usui 2007), environmental vertical wind shear (DeMaria 1996), and humidity in the midlevel troposphere (Emanuel et al. 2004). However, in an idealized condition where SSTs are fixed and the background vertical shear is absent, the intensity of a steady and axisymmetric TC can be derived by assuming the conservations of angular momentum and entropy, the gradient wind balance, and the hydrostatic balance (Emanuel 1986; Emanuel and Rotunno 2011):

$$
\left|V_{b}\right|^{2} \cong \frac{C_{k}}{C_{D}} \frac{T_{b}-T_{o}}{T_{s}}\left(k_{0}^{*}-k\right),
$$




\section{Case 2-4}

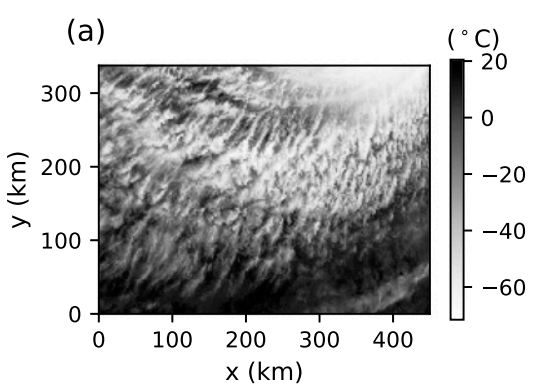

(b)

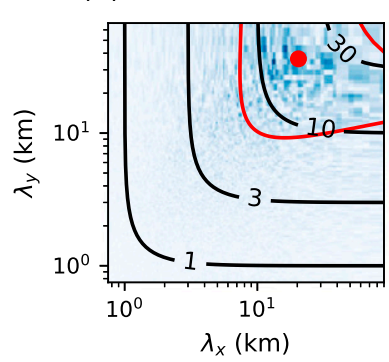

(c)

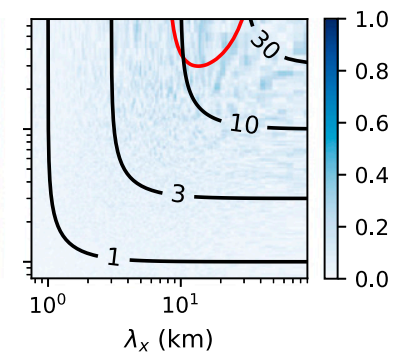

Case 2-5a

(d)

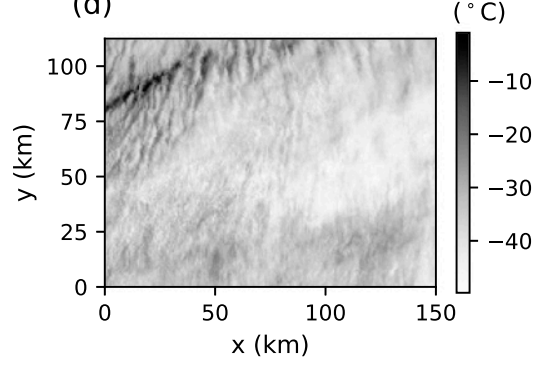

(g)
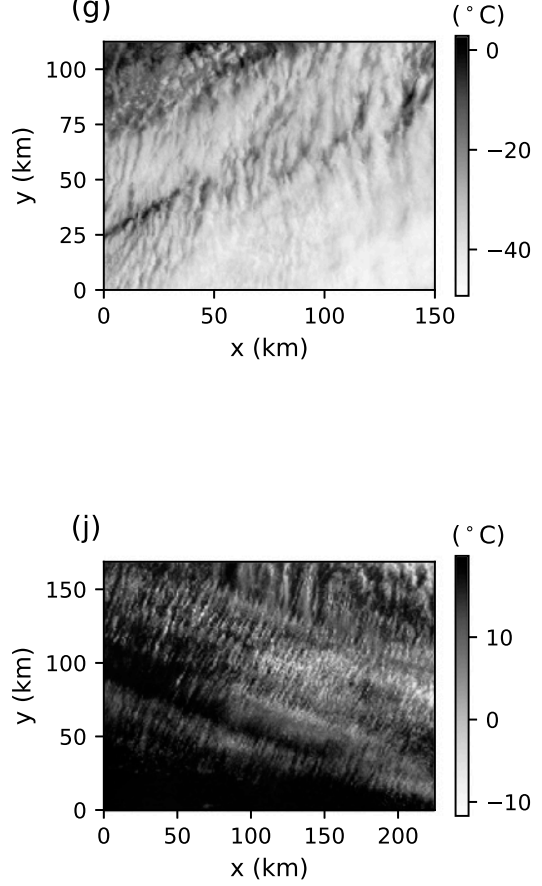

(e)

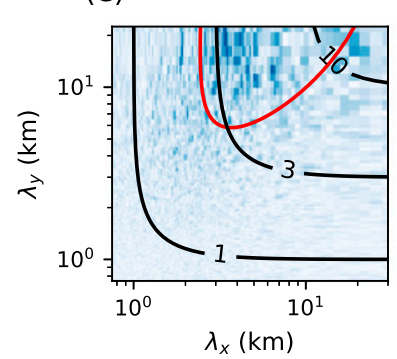

(f)

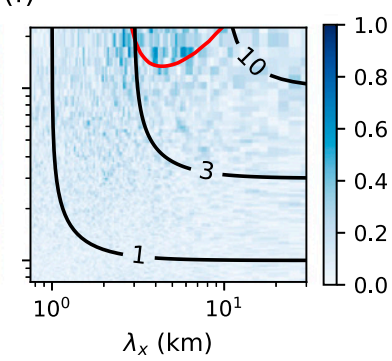

\section{Case 2-5b}

(h)

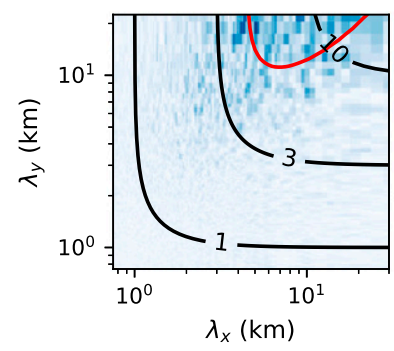

Case 2-6

(k)

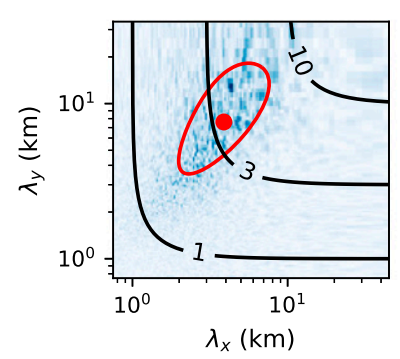

(i)

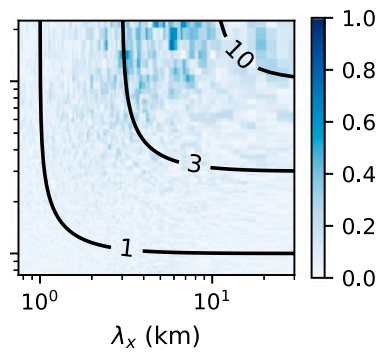

(I)

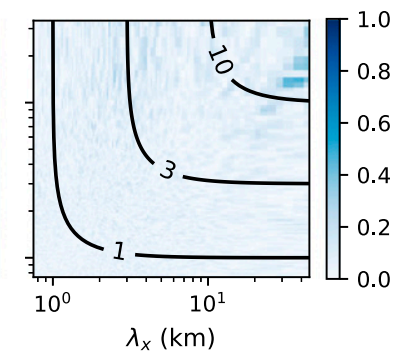

FIG. A2. As in Fig. 3, but for (a)-(c) case 2-4, (d)-(f) case 2-5a, (g)-(i) case 2-5b, and (j)-(l) case 2-6. 


\section{Case 3-1}

(a)

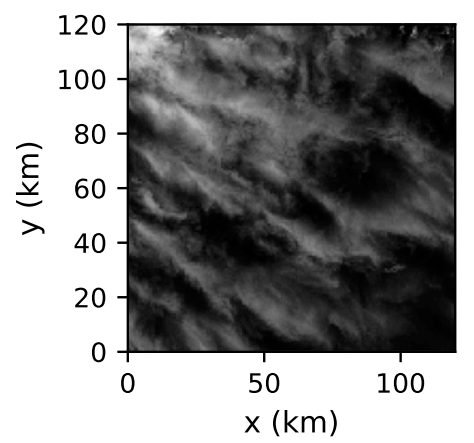

(d)

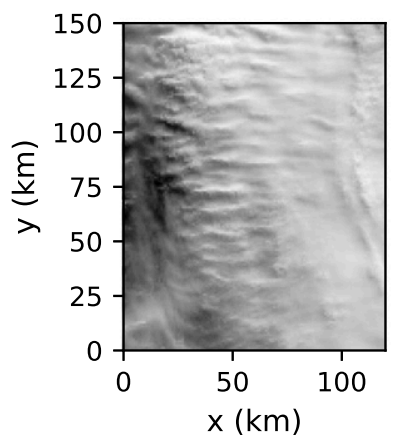

(b)

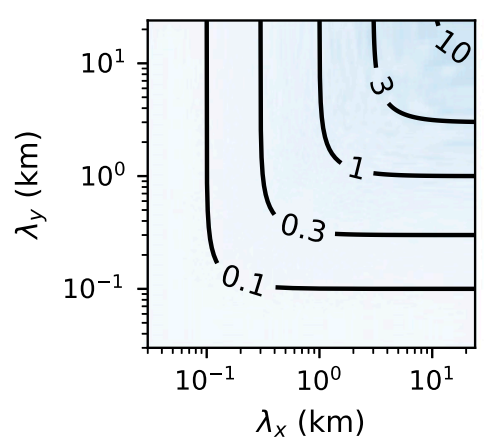

Case 3-2

(e)

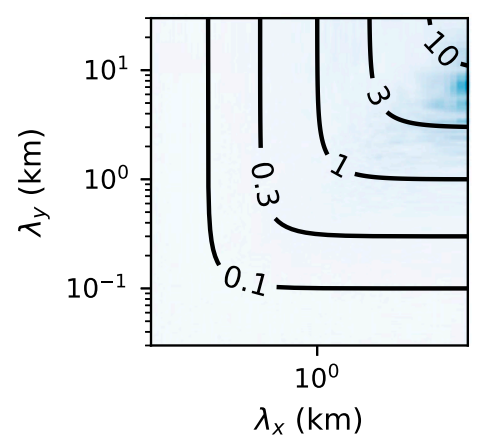

(c)

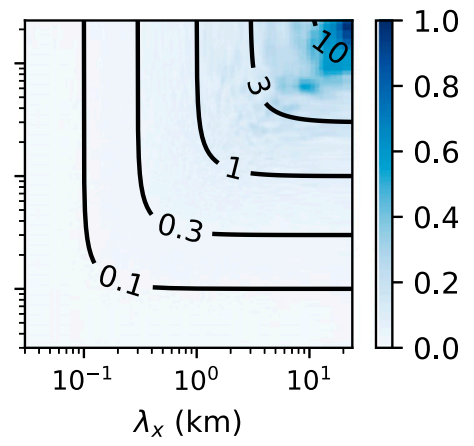

(f)

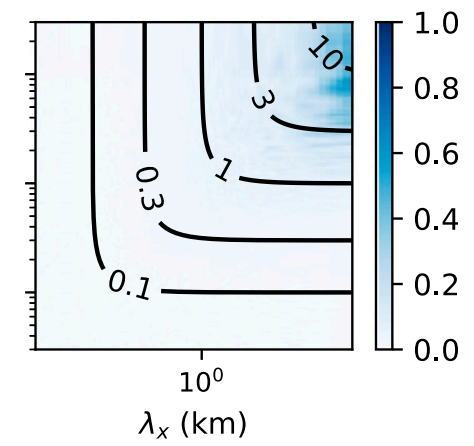

FIG. A3. As in Fig. 3, but for (a)-(c) case 3-1 and (d)-(f) case 3-2, and for shortwave radiances instead of infrared brightness temperatures. Red contours and dots indicating fitted parameters are now shown because the fitting was not conducted for those cases.

where $V_{b}$ is the speed of the gradient wind at the top of the boundary layer; $C_{k}$ and $C_{D}$ are dimensionless exchange coefficients for enthalpy and momentum, respectively; $T_{b}$ and $T_{o}$ are the air temperatures at the top of the boundary layer and in the outflow, respectively; $T_{s}$ is the sea surface temperature; $k_{0}^{*}$ is the saturation enthalpy of the sea surface; and $k$ is the enthalpy of the air just above the sea. Emanuel and Rotunno (2011) investigated the outflow temperature $T_{o}$ as a critical variable to determine the TC intensity using an axisymmetric model, indicating that $T_{o}$ is not simply determined by environmental conditions but is self-determined by the mixing processes inside the storm's core. Thus, they concluded that internal mixing processes can affect the intensity of a TC (Emanuel 2012). Although their model was incapable of representing cirrus bands due to its axisymmetric structure, mixing introduced by perturbations accompanying cirrus bands can affect $T_{o}$, modify heat budgets and, thus, potentially affect the intensities of TCs over a long time scale. Further understanding of the interaction between the outflow and cirrus banding is required to verify speculation on the importance of this possible multiscale interaction.

\section{Summary}

In this study, the structures and formation mechanisms of cirrus banding were investigated by radiosonde observations, high-resolution numerical experiments, and linear stability analyses. Previously, observational knowledge of thermal structures within cirrus bands has been limited. In this work, by analyzing 2 years of high-resolution radiosonde profiles around Japan, we found that a statically unstable layer exists in all of the 29 observed cases of vivid cirrus bands, and these bands are aligned nearly parallel to the vertical shear vector in the unstable layer. In high-resolution numerical experiments using a cloud-resolving model SCALE-RM, cirrus bands on anvil clouds of a TC are explicitly simulated in an idealized experiment. Statically unstable layers and band-parallel vertical wind shear were identified in these simulations. Sensitivity experiments and heat budget analyses demonstrate that the unstable stratification within cirrus clouds was maintained by the CRI, which heats the cloud bottom and cools the cloud top by approximately $0.5 \mathrm{~K} \mathrm{~h}^{-1}$. This result provides quantitative support for previous modeling studies (Trier et al. 2010; Kim et al. 2014; Trier and Sharman 2016). In the linear stability analyses, 
structures of growing perturbations were calculated on a basic state constructed based on radiosonde-observed profiles. We extended previous analyses in highly simplified basic states (Hill 1968; Asai 1970) or dry dynamics (Ohno and Miura 1982) using a relatively complex basic state constructed from radiosonde observations. We implicitly incorporated the effects of moist processes on the static stability in cloudy layers. Specifically, this was accomplished by partly replacing buoyancy frequencies with moist buoyancy frequencies. The fastest-growing disturbance is highly similar to that of the thermal-shear instability in an isolated unstable layer obtained by Hill (1968) and Asai (1970) and is consistent with radiosonde observations and numerical simulations. The results from these three different approaches consistently indicate that the thermal-shear instability maintained by the CRI is responsible for the formation of cirrus banding. This argument is not only consistent with recent modeling studies (Trier et al. 2010; Kim et al. 2014; Trier and Sharman 2016) but also evaluates the environmental conditions for cirrus banding based on a large number of cases, quantifies thermodynamic destabilization rates from CRI, and provides a theoretical support for thermal-shear instability as the organization mechanism for the bands in a complex environment. Remaining topics for future studies may include the development of objective case detection and analyses of observed cirrus bands, investigating a possible diurnal cycle of the frequency of cirrus bands due to varying solar flux, research on the nonlinear effect on the horizontal wavelength of cirrus bands, detailed investigations of the roles of KHI-like perturbations in producing fishbone-shaped cirrus bands featuring cross-shear waves overlapped on shear-parallel bands, and confirming speculation on the impact of mixing by cirrus bands on the overall thermodynamic budget of TCs.

Acknowledgments. We thank Dr. Hiroshi Niino and Dr. Masayuki Kawashima for fruitful discussions. We also thank two anonymous reviewers and Dr. David B. Mechem for their constructive comments, which helped significantly improve and clarify this manuscript. Himawari- 8 imagery data were provided by the Data Integration and Analysis System (DIAS) operated by The University of Tokyo, NICT ScienceCloud operated by the National Institute of Information and Communications Technology (NICT), and the P-Tree System by the Japan Aerospace Exploration Agency (JAXA). The High-Resolution Cloud Analysis Index products were supplied by Weather Map Co., Ltd. This research used the computational resources of the $\mathrm{K}$ computer provided by the RIKEN Center for Computational Science through the HPCI System Research project (Project ID: hp170323), the Cooperative Research Activities of Collaborative Use of the Computing Facility of the Atmosphere and Ocean Research Institute, The University of Tokyo, and the Simulation System at the Graduate School of Simulation Studies, University of Hyogo. Radiosonde observation data were downloaded from the Tokyo Global Information System Center operated by the JMA. This work was supported by JSPS KAKENHI Grants JP25287119, JP16H04048, JP20H05731, JP20H05729, and JP21J11266.

\section{APPENDIX}

\section{Additional Spectra of LEO Satellite Images}

The results of the satellite spectral analyses excluded in Fig. 3 are shown in Figs. A1-A3. As summarized in Table 3, these cases have dominant wavelengths $\lambda_{c}$ of $3-18 \mathrm{~km}$.

\section{REFERENCES}

Agee, E. M., 1975: Some inferences of eddy viscosity associated with instabilities in the atmosphere. J. Atmos. Sci., 32, 642-646, https:// doi.org/10.1175/1520-0469(1975)032<0642:SIOEVA >2.0.CO;2.

Asai, T., 1970: Stability of a plane parallel flow with variable vertical shear and unstable stratification. J. Meteor. Soc. Japan, 48, 129-139, https://doi.org/10.2151/jmsj1965.48.2_129.

— , and I. Nakasuji, 1973: On the stability of Ekman boundary layer flow with thermally unstable stratification. J. Meteor. Soc. Japan, 51, 29-42, https://doi.org/10.2151/jmsj1965.51.1_29.

Bessho, K., and Coauthors, 2016: An introduction to Himawari-8/9Japan's new-generation geostationary meteorological satellites. J. Meteor. Soc. Japan, 94, 151-183, https://doi.org/10.2151/ jmsj.2016-009.

Bolton, D., 1980: The computation of equivalent potential temperature. Mon. Wea. Rev., 108, 1046-1053, https://doi.org/ 10.1175/1520-0493(1980)108<1046:TCOEPT > 2.0.CO;2.

Bretherton, C. S., P. N. Blossey, and M. Khairoutdinov, 2005: An energy-balance analysis of deep convective self-aggregation above uniform SST. J. Atmos. Sci., 62, 4273-4292, https:// doi.org/10.1175/JAS3614.1.

Brown, D. P., 2011: Characteristics of atmospheric humidity derived from reanalyses and stable isotopic measurements from space. Ph.D. thesis, University of Colorado Boulder, 190 pp., https://core.ac.uk/download/pdf/54848824.pdf.

Cao, C., J. Xiong, S. Blonski, Q. Liu, S. Uprety, X. Shao, Y. Bai, and F. Weng, 2013: Suomi NPP VIIRS sensor data record verification, validation, and long-term performance monitoring. J. Geophys. Res. Atmos., 118, 11 664-11 678, https://doi.org/ 10.1002/2013JD020418.

Chapman, C., and M. Proctor, 1980: Nonlinear Rayleigh-Bénard convection between poorly conducting boundaries. J. Fluid Mech., 101, 759-782, https://doi.org/10.1017/S0022112080001917.

DeMaria, M., 1996: The effect of vertical shear on tropical cyclone intensity change. J. Atmos. Sci., 53, 2076-2088, https://doi.org/ 10.1175/1520-0469(1996)053<2076:TEOVSO > 2.0.CO;2.

_ mum intensity of Atlantic tropical cyclones. J. Climate, 7, 1324-1334, https://doi.org/10.1175/1520-0442(1994)007<1324: SSTATM $>2.0 . \mathrm{CO} ; 2$.

Duran, P., and J. Molinari, 2016: Upper-tropospheric low Richardson number in tropical cyclones: Sensitivity to cyclone intensity and the diurnal cycle. J. Atmos. Sci., 73, 545554, https://doi.org/10.1175/JAS-D-15-0118.1.

$\longrightarrow$, and - 2019: Tropopause evolution in a rapidly intensifying tropical cyclone: A static stability budget analysis in an idealized axisymmetric framework. J. Atmos. Sci., 76, 209-229, https://doi.org/10.1175/JAS-D-18-0097.1.

Durran, D. R., and J. B. Klemp, 1982: On the effects of moisture on the Brunt-Väisälä frequency. J. Atmos. Sci., 39, 2152-2158, https://doi.org/10.1175/1520-0469(1982)039<2152:OTEOMO> 2.0.CO;2.

Ellrod, G. P., 1985: Detection of high level turbulence using satellite imagery and upper air data. NOAA Tech. Memo. NESDIS 10, 30 pp. 
Emanuel, K. A., 1986: An air-sea interaction theory for tropical cyclones. Part I: Steady-state maintenance. J. Atmos. Sci., 43, 585-605, https://doi.org/10.1175/1520-0469(1986) $043<0585$ :AASITF $>2.0 . \mathrm{CO} ; 2$.

_ 2012: Self-stratification of tropical cyclone outflow. Part II: Implications for storm intensification. J. Atmos. Sci., 69, 988996, https://doi.org/10.1175/JAS-D-11-0177.1.

—_, and R. Rotunno, 2011: Self-stratification of tropical cyclone outflow. Part I: Implications for storm structure. J. Atmos. Sci., 68, 2236-2249, https://doi.org/10.1175/JASD-10-05024.1.

— C. DesAutels, C. Holloway, and R. Korty, 2004: Environmental control of tropical cyclone intensity. J. Atmos. Sci., 61, 843-858, https://doi.org/10.1175/1520-0469(2004)061<0843:ECOTCI> 2.0.CO;2.

Glickman, T., Ed., 2000: Glossary of Meteorology. 2nd ed. Amer. Meteor. Soc., 855 pp., http://glossary.ametsoc.org/.

Hersbach, H., and Coauthors, 2020: The ERA5 global reanalysis. Quart. J. Roy. Meteor. Soc., 146, 1999-2049, https://doi.org/ 10.1002/qj.3803.

Hill, G. E., 1968: On the orientation of cloud bands. Tellus, 20, 132-137, https://doi.org/10.3402/tellusa.v20i1.9936.

Howard, L. N., 1961: Note on a paper of John W. Miles. J. Fluid Mech., 10, 509-512, https://doi.org/10.1017/S0022112061000317.

Jaubert, G., C. Piriou, S. M. Loehrer, A. Petitpa, and J. A. Moore, 1999: Development and quality control of the FASTEX data archive. Quart. J. Roy. Meteor. Soc., 125, 3165-3188, https:// doi.org/10.1002/qj.49712556104.

Kain, J. S., and J. M. Fritsch, 1989: A one-dimensional entraining/detraining plume model and its application in convective parameterization. J. Atmos. Sci., 47, 27842802, https://doi.org/10.1175/1520-0469(1990)047<2784: AODEPM $>2.0 . \mathrm{CO} ; 2$.

Kim, J., H. Chun, R. D. Sharman, and S. B. Trier, 2014: The role of vertical shear on aviation turbulence within cirrus bands of a simulated western Pacific cyclone. Mon. Wea. Rev., 142, 27942813, https://doi.org/10.1175/MWR-D-14-00008.1.

Kizu, N., T. Sugidachi, E. Kobayashi, S. Hshino, K. Shimizu, R. Maeda, and M. Fujiwara, 2018: Technical characteristics and GRUAN data processing for the Meisei RS-11G and iMS-100 radiosondes. GRUAN Tech. Doc. GRUAN-TD-5, 152 pp., https://www.gruan.org/documentation/gruan/td/gruan-td-5.

Knox, J. A., A. S. Bachmeier, W. M. Carter, J. E. Tarantino, L. C. Paulik, E. N. Wilson, G. S. Bechdol, and M. J. Mays, 2010: Transverse cirrus bands in weather systems: A grand tour of an enduring enigma. Weather, 65, 35-41, https://doi.org/10.1002/wea.417.

Korolev, A., and G. A. Isaac, 2006: Relative humidity in liquid, mixed-phase, and ice clouds. J. Atmos. Sci., 63, 2865-2880, https://doi.org/10.1175/JAS3784.1.

Krishnamurti, R., 1968: Finite amplitude convection with changing mean temperature. Part 1. Theory. J. Fluid Mech., 33, 445-455, https://doi.org/10.1017/S0022112068001436.

LeMone, M. A., 1973: The structure and dynamics of horizontal roll vortices in the planetary boundary layer. J. Atmos. Sci., 30, 1077-1091, https://doi.org/10.1175/1520-0469(1973)030<1077: TSADOH $>2.0 . \mathrm{CO} ; 2$.

Lenz, A., K. M. Bedka, W. F. Feltz, and S. A. Ackerman, 2009: Convectively induced transverse band signatures in satellite imagery. Wea. Forecasting, 24, 1362-1373, https://doi.org/ 10.1175/2009WAF2222285.1.

Mellor, G. L., and T. Yamada, 1982: Development of a turbulence closure model for geophysical fluid problems. Rev. Geophys., 20, 851-875, https://doi.org/10.1029/RG020i004p00851.
Miles, J. W., 1961: On the stability of heterogeneous shear flows. J. Fluid Mech., 10, 496-508, https://doi.org/10.1017/ S0022112061000305.

Miller, J., U. Nair, R. Ramachandran, and M. Maskey, 2018: Detection of transverse cirrus bands in satellite imagery using deep learning. Comput. Geosci., 118, 79-85, https://doi.org/ 10.1016/j.cageo.2018.05.012.

Molinari, J., P. Duran, and D. Vollaro, 2014: Low Richardson number in the tropical cyclone outflow layer. J. Atmos. Sci., 71, 3164-3179, https://doi.org/10.1175/JAS-D-14-0005.1.

Nakanishi, M., and H. Niino, 2004: An improved Mellor-Yamada level-3 model with condensation physics: Its design and verification. Bound.-Layer Meteor., 112, 1-31, https://doi.org/ 10.1023/B:BOUN.0000020164.04146.98.

Ninomiya, K., 2010: Intense rainfalls on August 17, 1968 over the Kiso-Hida and Nagara River basin in Japan associated with intrusion of middle tropospheric dry airs over the low-level moist belt. J. Meteor. Soc. Japan, 88, 737-754, https://doi.org/ 10.2151/jmsj.2010-406.

Nishizawa, S., H. Yashiro, Y. Sato, Y. Miyamoto, and H. Tomita, 2015: Influence of grid aspect ratio on planetary boundary layer turbulence in large-eddy simulations. Geosci. Model Dev., 8, 3393-3419, https://doi.org/10.5194/gmd-8-33932015.

, and Coauthors, 2018: Detailed formulation of SCALE-RM. SCALE Rep., 136 pp., https://scale.riken.jp/archives/scale_ rm_description-5.4.4.pdf.

Nolan, D. S., E. D. Rappin, and K. A. Emanuel, 2007: Tropical cyclogenesis sensitivity to environmental parameters in radiative-convective equilibrium. Quart. J. Roy. Meteor. Soc., 133, 2085-2107, https://doi.org/10.1002/qj.170.

Ohno, H., and N. Miura, 1982: Excitation of Kelvin-Helmholtz waves beneath the tropopause: Relation to transverse cirrus bands. Tenki, 29, 1235-1241.

Roy, D., and Coauthors, 2014: Landsat-8: Science and product vision for terrestrial global change research. Remote Sens. Environ., 145, 154-172, https://doi.org/10.1016/ j.rse.2014.02.001.

Sato, Y., S. Nishizawa, H. Yashiro, Y. Miyamoto, Y. Kajikawa, and H. Tomita, 2015: Impacts of cloud microphysics on trade wind cumulus: Which cloud microphysics processes contribute to the diversity in a large eddy simulation? Prog. Earth Planet. Sci., 2, 23, https://doi.org/10.1186/s40645-0150053-6.

Sekiguchi, M., and T. Nakajima, 2008: A $k$-distribution-based radiation code and its computational optimization for an atmospheric general circulation model. J. Quant. Spectrosc. Radiat. Transfer, 109, 2779-2793, https://doi.org/10.1016/ j.jqsrt.2008.07.013.

Suzue, H., T. Imai, and K. Mouri, 2016: High-resolution cloud analysis information derived from Himawari-8 data. Meteorological Satellite Center Tech. Note 61, 43-51, https://www.data.jma.go.jp/mscweb/technotes/ msctechrep61-4.pdf.

Tomita, H., 2008: New microphysical schemes with five and six categories by diagnostic generation of cloud ice. J. Meteor. Soc. Japan, 86A, 121-142, https://doi.org/10.2151/jmsj.86A.121.

Trier, S. B., and R. D. Sharman, 2016: Mechanisms influencing cirrus banding and aviation turbulence near a convectively enhanced upper-level jet stream. Mon. Wea. Rev., 144, 3003-3027, https:// doi.org/10.1175/MWR-D-16-0094.1.

,$\ldots$, R. G. Fovell, and R. G. Frehlich, 2010: Numerical simulation of radial cloud bands within the upper-level outflow of 
an observed mesoscale convective system. J. Atmos. Sci., 67, 2990-2999, https://doi.org/10.1175/2010JAS3531.1.

Wada, A., and N. Usui, 2007: Importance of tropical cyclone heat potential for tropical cyclone intensity and intensification in the western North Pacific. J. Oceanogr., 63, 427-447, https:// doi.org/10.1007/s10872-007-0039-0.

Weckwerth, T. M., J. W. Wilson, R. M. Wakimoto, and N. A. Crook, 1997: Horizontal convective rolls: Determining the environmental conditions supporting their existence and characteristics.
Mon. Wea. Rev., 125, 505-526, https://doi.org/10.1175/15200493(1997)125<0505:HCRDTE>2.0.CO;2.

$\mathrm{Xu}$, J., and Y. Wang, 2018: Effect of the initial vortex structure on intensification of a numerically simulated tropical cyclone. J. Meteor. Soc. Japan, 96, 111-126, https://doi.org/10.2151/jmsj.2018-014.

Zehr, R., 2004: Satellite products and imagery with Hurricane Isabel. Proc. 13th Conf. on Satellite Meteorology and Oceanography, Norfolk, VA, Amer. Meteor. Soc., P5.1, http://ams.confex.com/ ams/pdfpapers/79158.pdf. 